\title{
Electrochemical Studies of Verdazyl Radicals
}

\author{
Joe B. Gilroy, Stephen D. J. McKinnon, Bryan D. Koivisto, and Robin G. Hicks*
}

Department of Chemistry, University of Victoria, PO Box 3065, Victoria, BC, Canada, V8W 3V6.

rhicks@uvic.ca

\section{SUPPORTING INFORMATION}

\section{CONTENTS}

$\begin{array}{ll}\text { Experimental Details and Characterization Data } & \text { S2 }\end{array}$

${ }^{1} \mathrm{H}$ and ${ }^{13} \mathrm{C}$ NMR spectra $\quad$ S9

CV Data $\quad$ S33

$\begin{array}{ll}\text { EPR Data } & \text { S46 }\end{array}$

$\begin{array}{ll}\text { References } & \text { S50 }\end{array}$ 


\section{Experimental}

\section{General Considerations}

All reactions and manipulations were carried out under an argon atmosphere using standard Schlenk or glovebox techniques unless stated otherwise. Solvents were dried and distilled under argon prior to use. All reagents were purchased from Aldrich and used as received. Formazans were prepared via literature methods. ${ }^{1}$ Formazans $4 \mathbf{4 a},{ }^{1} \mathbf{c},{ }^{2} \mathbf{d},{ }^{3} \mathbf{g},{ }^{4} \mathbf{h}^{3}$ and verdazyl radicals $1 \mathbf{a},{ }^{5} \mathbf{c},{ }^{2} \mathbf{d},{ }^{6} \mathbf{g},{ }^{7} \mathbf{h}^{6}$ have been reported previously, however, with the exception of compounds $\mathbf{4 d}$ and $\mathbf{4 h}$ their characterization was incomplete or they were produced via different synthetic routes, prompting us to report previously overlooked characterization details. Verdazyls 1a-e, g-i were prepared via literature methods. ${ }^{8}$ Verdazyls $\mathbf{2 b},{ }^{9} \mathbf{2 c},{ }^{10} \mathbf{2 d},{ }^{11}$ and $\mathbf{2} \mathbf{e}^{11}$ were prepared according to literature methods and their characterization assumed sufficient. NMR spectra were recorded on a $300 \mathrm{MHz}$ Bruker instrument, ${ }^{13} \mathrm{C}$ shifts at $\sim 108$ ppm were ignored as they are commonly observed as artifacts of the experiment on this instrument. Infrared spectra were recorded as $\mathrm{KBr}$ pellets using a Perkin Elmer Spectrum One instrument. UV-Vis spectra were recorded using a Cary 50 Scan instrument. EPR spectra were recorded on a Bruker EMX EPR instrument equipped with an X-band microwave bridge. Cyclic voltammetry experiments were performed with a Bioanalytical Systems CV50 voltammetric analyzer. Typical electrochemical cells consisted of a three-electrode setup including a glassy carbon working electrode, platinum counter electrode, and silver reference. Experiments were run at a scan rate of 100 $\mathrm{mV} / \mathrm{s}$. Acetonitrile solutions of analyte $(\sim 1 \mathrm{mM})$ and electrolyte $\left(0.1 \mathrm{M} \mathrm{Bu}_{4} \mathrm{~N}^{+} \mathrm{BF}_{4}^{-}\right)$were referenced against an internal standard $(\sim 1 \mathrm{mM}$ Fc). Elemental analyses were carried out by Canadian Microanalytical Services Ltd., Vancouver, BC.

\section{Synthesis}

General procedure for formazans 4a-j, l,m : 1,3,5-triphenylformazan (4a). In a typical procedure, phenylhydrazine $(3.54 \mathrm{~g}, 33 \mathrm{mmol})$ was combined with ethanol $(50 \mathrm{~mL})$ and benzaldehyde $(3.50 \mathrm{~g}, 33$ mmol). After stirring for $2 \mathrm{~h}$ a yellow crystalline precipitate had formed. The reaction mixture was treated with sodium carbonate hydrate $(16.5 \mathrm{~g}, 133 \mathrm{mmol})$, tetrabutylammonium bromide $(1.25 \mathrm{~g}, 4$ 
mmol), water $(60 \mathrm{~mL})$, and dichloromethane $(100 \mathrm{~mL})$ before being stirred at $0^{\circ} \mathrm{C}$ for $1 \mathrm{~h}$. A solution of diazonium salt made from stirring aniline $(3.52 \mathrm{~g}, 38 \mathrm{mmol})$, sodium nitrite $(3.15 \mathrm{~g}, 46 \mathrm{mmol})$, water $(10 \mathrm{~mL})$, and hydrochloric acid $(10 \mathrm{~mL})$ for $30 \mathrm{~min}$ at $0^{\circ} \mathrm{C}$ was then added dropwise. Upon addition, the organic phase in the biphasic reaction mixture turned blood red. After stirring for $2 \mathrm{~h}$ at RT the organic layer was collected, washed with water $(3 \times 100 \mathrm{~mL})$ and taken to dryness in vacuo. Column chromatography (neutral alumina, dichloromethane) followed by trituration of the solid with methanol afforded 4a as a dark purple microcrystalline solid, yield $6.50 \mathrm{~g}(65.6 \%) . \quad \mathrm{Mp} . \quad 148-150^{\circ} \mathrm{C} .{ }^{1} \mathrm{H} \mathrm{NMR}$ $\left(\mathrm{CD}_{2} \mathrm{Cl}_{2}\right): \delta 15.36(\mathrm{~s}, 1 \mathrm{H}), 8.16\left(\mathrm{~d}, 2 \mathrm{H},{ }^{3} \mathrm{~J}=8 \mathrm{~Hz}\right), 7.73\left(\mathrm{~d}, 4 \mathrm{H},{ }^{3} \mathrm{~J}=8 \mathrm{~Hz}\right), 7.60-7.25(\mathrm{~m}, 9 \mathrm{H}) \mathrm{ppm} .{ }^{13} \mathrm{C}$ $\operatorname{NMR}\left(\mathrm{CD}_{2} \mathrm{Cl}_{2}\right): \delta 148.5,141.7,138.0,130.4,130.0,129.7,129.6,129.5,129.0,128.4,128.2,128.1$, 126.4, 122.7, 119.3 ppm. FT-IR (KBr): 1597 (s), 1510 (s), 1492 (s), 1232 (s), 1017 (s), 752 (s) cm cm $^{-1}$ UV-Vis $\left(\mathrm{CH}_{2} \mathrm{Cl}_{2}\right): \lambda_{\max } 270 \mathrm{~nm}(\varepsilon=17250), 300 \mathrm{~nm}(\varepsilon=22000), 488 \mathrm{~nm}(\varepsilon=14250) . \mathrm{MS}(\mathrm{EI}): \mathrm{m} / z 300$ $\left(\mathrm{M}^{+}, 35 \%\right)$. Anal. Calcld for $\mathrm{C}_{19} \mathrm{H}_{16} \mathrm{~N}_{4}: \mathrm{C}, 75.98 ; \mathrm{H}, 5.37 ; \mathrm{N}, 18.65$. Found: $\mathrm{C}, 76.16 ; \mathrm{H}, 5.54 ; \mathrm{N}$, 18.36.

1,5-di-p-methoxyphenyl-3-p-tolylformazan (4b). $\quad$ Yield 26.2 \%. $\quad$ Mp. $\quad 131-133^{\circ} \mathrm{C} . \quad{ }^{1} \mathrm{H}$ NMR $\left(\mathrm{CD}_{2} \mathrm{Cl}_{2}\right): \delta 15.40(\mathrm{~s}, 1 \mathrm{H}), 8.01\left(\mathrm{~d}, 2 \mathrm{H},{ }^{3} \mathrm{~J}=8 \mathrm{~Hz}\right), 7.66\left(\mathrm{~d}, 4 \mathrm{H},{ }^{3} \mathrm{~J}=9 \mathrm{~Hz}\right), 7.25\left(\mathrm{~d}, 2 \mathrm{H},{ }^{3} \mathrm{~J}=8 \mathrm{~Hz}\right), 7.00$ $\left(\mathrm{d}, 4 \mathrm{H},{ }^{3} \mathrm{~J}=9 \mathrm{~Hz}\right), 3.86(\mathrm{~s}, 6 \mathrm{H}), 2.41(\mathrm{~s}, 3 \mathrm{H}) \mathrm{ppm} .{ }^{13} \mathrm{C} \mathrm{NMR}\left(\mathrm{CD}_{2} \mathrm{Cl}_{2}\right): \delta 159.8,142.5,137.7,129.6$, 126.1, 120.5, 115.2, 56.2, 21.5 ppm. FT-IR (KBr): 1599 (s), 1507 (s), 1255 (s), 1230 (s), 1033 (s), 819 (s) $\mathrm{cm}^{-1}$. UV-Vis $\left(\mathrm{CH}_{2} \mathrm{Cl}_{2}\right): \lambda_{\max } 314 \mathrm{~nm}(\varepsilon=20750), 538 \mathrm{~nm}(\varepsilon=17000) . \mathrm{MS}(\mathrm{EI}): m / z 374\left(\mathrm{M}^{+}, 30\right.$ \%). Anal. Calcld for $\mathrm{C}_{22} \mathrm{H}_{22} \mathrm{~N}_{4} \mathrm{O}_{2}$ : C, 70.57; H, 5.92; N, 14.96. Found: C, 70.19; H, 6.16; N, 15.08.

1,3,5-tri-p-tolylformazan (4c). Yield 56.7 \%. Mp. $170-172{ }^{\circ} \mathrm{C} .{ }^{1} \mathrm{H} \mathrm{NMR}\left(\mathrm{CD}_{2} \mathrm{Cl}_{2}\right): \delta 15.35(\mathrm{~s}, 1 \mathrm{H})$, $8.02\left(\mathrm{~d}, 2 \mathrm{H},{ }^{3} \mathrm{~J}=8 \mathrm{~Hz}\right), 7.61\left(\mathrm{~d}, 4 \mathrm{H},{ }^{3} \mathrm{~J}=8 \mathrm{~Hz}\right), 7.35-7.25\left(\mathrm{~m}, 6 \mathrm{H},{ }^{3} \mathrm{~J}=8 \mathrm{~Hz}\right), 2.40(\mathrm{~s}, 9 \mathrm{H}) \mathrm{ppm} .{ }^{13} \mathrm{C}$ NMR $\left(\mathrm{CD}_{2} \mathrm{Cl}_{2}\right): \delta 146.4,141.5,138.3,138.2,137.9,135.4,130.6,129.6,126.2,119.1,21.5$ ppm. FT-IR (KBr): $1598(\mathrm{~m}), 1508(\mathrm{~s}), 1226(\mathrm{~s}), 816(\mathrm{~s}) \mathrm{cm}^{-1}$. UV-Vis $\left(\mathrm{CH}_{2} \mathrm{Cl}_{2}\right): \lambda_{\max } 279 \mathrm{~nm}(\varepsilon=19000), 307 \mathrm{~nm}$ ( $\varepsilon=26750), 498 \mathrm{~nm}(17000)$. MS (EI): $\mathrm{m} / z 342\left(\mathrm{M}^{+}, 100 \%\right)$. Anal. Calcld for $\mathrm{C}_{22} \mathrm{H}_{22} \mathrm{~N}_{4}: \mathrm{C}, 77.16 ; \mathrm{H}$, 6.48; N, 16.36. Found: C, 76.66; H, 6.51; N, 16.04. 
1,5-di-p-chlorophenyl-3-p-tolylformazan (4e). Yield 61.5 \%. Mp. 198-200 ${ }^{\circ} \mathrm{C} .{ }^{1} \mathrm{H}$ NMR $\left(\mathrm{CD}_{2} \mathrm{Cl}_{2}\right): \delta$ $15.07(\mathrm{~s}, 1 \mathrm{H}), 7.98\left(\mathrm{~d}, 2 \mathrm{H},{ }^{3} \mathrm{~J}=8 \mathrm{~Hz}\right), 7.65\left(\mathrm{~d}, 4 \mathrm{H},{ }^{3} \mathrm{~J}=9 \mathrm{~Hz}\right), 7.45\left(\mathrm{~d}, 4 \mathrm{H},{ }^{3} \mathrm{~J}=9 \mathrm{~Hz}\right), 7.26\left(\mathrm{~d}, 2 \mathrm{H},{ }^{3} \mathrm{~J}=\right.$ $8 \mathrm{~Hz}), 2.41(\mathrm{~s}, 3 \mathrm{H}) \mathrm{ppm} .{ }^{13} \mathrm{C} \mathrm{NMR}\left(\mathrm{CD}_{2} \mathrm{Cl}_{2}\right): \delta 147.1,142.2,138.5,134.7,133.3,130.1,129.7,126.4$, 120.5, 21.5 ppm. FT-IR (KBr): 1509 (s), 1488 (s), 1479 (s), 1406 (s), 818 (s) cm ${ }^{-1}$. UV-Vis $\left(\mathrm{CH}_{2} \mathrm{Cl}_{2}\right)$ : $\lambda_{\max } 303 \mathrm{~nm}(\varepsilon=28250), 503 \mathrm{~nm}(\varepsilon=17000) . \mathrm{MS}(\mathrm{EI}): m / z 382\left(\mathrm{M}^{+}, 10 \%\right)$. Anal. Calcld for $\mathrm{C}_{20} \mathrm{H}_{16} \mathrm{Cl}_{2} \mathrm{~N}_{4}: \mathrm{C}, 62.67 ; \mathrm{H}, 4.21 ; \mathrm{N}, 14.62$. Found: C, 62.67; H, 4.29; N, 14.65.

1,5-di-p-cyanophenyl-3-p-tolylformazan (4f). Yield 43.3 \%. Mp. 214-216 ${ }^{\circ}$. ${ }^{1} \mathrm{H}$ NMR $\left(\mathrm{CD}_{2} \mathrm{Cl}_{2}\right): \delta$ $14.69(\mathrm{~s}, 1 \mathrm{H}), 7.97\left(\mathrm{~d}, 2 \mathrm{H},{ }^{3} \mathrm{~J}=8 \mathrm{~Hz}\right), 7.78(\mathrm{~s}(\mathrm{br}), 8 \mathrm{H}), 7.29\left(\mathrm{~d}, 2 \mathrm{H},{ }^{3} \mathrm{~J}=8 \mathrm{~Hz}\right), 2.42(\mathrm{~s}, 3 \mathrm{H}) \mathrm{ppm} .{ }^{13} \mathrm{C}$ $\operatorname{NMR}\left(\mathrm{CD}_{2} \mathrm{Cl}_{2}\right): \delta 151.1,143.8,139.5,134.2,133.8,133.7,130.9,129.9,126.8,123.7,119.8,119.2$ 115.1, 111.1, 21.6 ppm. FT-IR (KBr): 2223 (s), 1509 (s), 1223 (s) cm ${ }^{-1}$. UV-Vis $\left(\mathrm{CH}_{2} \mathrm{Cl}_{2}\right): \lambda_{\max } 297 \mathrm{~nm}$ $(\varepsilon=46250), 513 \mathrm{~nm}(\varepsilon=17250) . \mathrm{MS}(\mathrm{EI}): m / z 364\left(\mathrm{M}^{+}, 15 \%\right)$. Anal. Calcld for $\mathrm{C}_{22} \mathrm{H}_{16} \mathrm{~N}_{6}: \mathrm{C}, 72.51$; H, 4.43; N, 23.06. Found: C, 72.49; H, 4.49; N, 23.19.

3-p-methoxyphenyl-1,5-di-p-tolylformazan (4g). $\quad$ Yield $59.5 \%$ \%. $\quad$ Mp. $\quad 162-164^{\circ} \mathrm{C} . \quad{ }^{1} \mathrm{H}$ NMR $\left(\mathrm{CD}_{2} \mathrm{Cl}_{2}\right): \delta 15.24(\mathrm{~s}, 1 \mathrm{H}), 8.06\left(\mathrm{~d}, 2 \mathrm{H},{ }^{3} \mathrm{~J}=9 \mathrm{~Hz}\right), 7.60\left(\mathrm{~d}, 4 \mathrm{H},{ }^{3} \mathrm{~J}=8 \mathrm{~Hz}\right), 7.28\left(\mathrm{~d}, 4 \mathrm{H},{ }^{3} \mathrm{~J}=8 \mathrm{~Hz}\right), 6.98$ $\left(\mathrm{d}, 2 \mathrm{H},{ }^{3} \mathrm{~J}=9 \mathrm{~Hz}\right), 3.87(\mathrm{~s}, 3 \mathrm{H}), 2.40(\mathrm{~s}, 6 \mathrm{H}) \mathrm{ppm} .{ }^{13} \mathrm{C} \mathrm{NMR}\left(\mathrm{CD}_{2} \mathrm{Cl}_{2}\right): \delta 166.1,160.0,154.0,146.5$ 141.1, 138.1, 130.9, 130.6, 127.6, 119.1, 114.2, 55.8, 21.5 ppm. FT-IR (KBr): 1605 (m), 1508 (s), 1250 $(\mathrm{s}), 1222(\mathrm{~s}), 1025(\mathrm{~s}), 810(\mathrm{~m}) \mathrm{cm}^{-1}$. UV-Vis $\left(\mathrm{CH}_{2} \mathrm{Cl}_{2}\right): \lambda_{\max } 279 \mathrm{~nm}(\varepsilon=20750), 308 \mathrm{~nm}(\varepsilon=27500)$ $522 \mathrm{~nm}(\varepsilon=16000) . \mathrm{MS}(\mathrm{EI}): m / z 358\left(\mathrm{M}^{+}, 20 \%\right)$. Anal. Calcld for $\mathrm{C}_{22} \mathrm{H}_{22} \mathrm{~N}_{4} \mathrm{O}: \mathrm{C}, 73.72 ; \mathrm{H}, 6.19 ; \mathrm{N}$, 15.63. Found: C, 73.77; H, 6.22; N, 15.71 .

3-p-chlorophenyl-1,5-di-p-tolylformazan (4i). Yield $43.7 \%$. Mp. $188-190^{\circ} \mathrm{C} .{ }^{1} \mathrm{H}$ NMR $\left(\mathrm{CD}_{2} \mathrm{Cl}_{2}\right): \delta$ $15.40(\mathrm{~s}, 1 \mathrm{H}), 8.00\left(\mathrm{~d}, 2 \mathrm{H},{ }^{3} \mathrm{~J}=9 \mathrm{~Hz}\right), 7.51\left(\mathrm{~d}, 4 \mathrm{H},{ }^{3} \mathrm{~J}=8 \mathrm{~Hz}\right), 7.31\left(\mathrm{~d}, 2 \mathrm{H},{ }^{3} \mathrm{~J}=9 \mathrm{~Hz}\right), 7.19\left(\mathrm{~d}, 4 \mathrm{H},{ }^{3} \mathrm{~J}=\right.$ $8 \mathrm{~Hz}), 2.31(\mathrm{~s}, 6 \mathrm{H}) \mathrm{ppm} .{ }^{13} \mathrm{C} \mathrm{NMR}\left(\mathrm{CD}_{2} \mathrm{Cl}_{2}\right): \delta 146.2,140.3,138.6,136.9,133.6,130.6,129.0,127.5$, 119.2, 21.5 ppm. FT-IR (KBr): $1488(\mathrm{~s}), 1396(\mathrm{~m}), 1238(\mathrm{~s}) \mathrm{cm}^{-1} . \mathrm{UV}-\mathrm{Vis}\left(\mathrm{CH}_{2} \mathrm{Cl}_{2}\right): \lambda_{\max } 235 \mathrm{~nm}(\varepsilon=$ 16750), $274 \mathrm{~nm}(\varepsilon=18000), 313 \mathrm{~nm}(\varepsilon=31250), 496 \mathrm{~nm}(\varepsilon=20000) . \mathrm{MS}(\mathrm{EI}): \mathrm{m} / z 362\left(\mathrm{M}^{+}, 10 \%\right)$ Anal. Calcld for $\mathrm{C}_{21} \mathrm{H}_{19} \mathrm{ClN}_{4}$ : C, 69.51; H, 5.28; N, 15.44. Found: C, 69.63; H, 5.25; N, 15.63. 
3-p-cyanophenyl-1,5-di-p-tolylformazan (4j). Yield 30.1\%. Mp. 204-206 ${ }^{\circ}$. ${ }^{1} \mathrm{H}$ NMR $\left(\mathrm{CD}_{2} \mathrm{Cl}_{2}\right): \delta$ 15.60 (s (br), 1H), $8.16\left(\mathrm{~d}, 2 \mathrm{H},{ }^{3} \mathrm{~J}=9 \mathrm{~Hz}\right), 7.61\left(\mathrm{~d}, 2 \mathrm{H},{ }^{3} \mathrm{~J}=9 \mathrm{~Hz}\right), 7.51\left(\mathrm{~d}, 2 \mathrm{H},{ }^{3} \mathrm{~J}=8 \mathrm{~Hz}\right), 7.20(\mathrm{~d}, 2 \mathrm{H}$, $\left.{ }^{3} \mathrm{~J}=8 \mathrm{~Hz}\right), 2.32(\mathrm{~s}, 6 \mathrm{H}) \mathrm{ppm} .{ }^{13} \mathrm{C} \mathrm{NMR}\left(\mathrm{CD}_{2} \mathrm{Cl}_{2}\right): \delta 145.9,142.7,139.5,139.1,132.8,130.7,126.2$, 119.9, 119.4, 110.8, 21.6 ppm. FT-IR (KBr): 2221 (s), 1601 (s), 1498 (s), 1249 (s) cm ${ }^{-1}$. UV-Vis $\left(\mathrm{CH}_{2} \mathrm{Cl}_{2}\right): \lambda_{\max } 272 \mathrm{~nm}(\varepsilon=49250), 339 \mathrm{~nm}(\varepsilon=34000), 488 \mathrm{~nm}(\varepsilon=23000) . \mathrm{MS}(\mathrm{EI}): m / z 353\left(\mathrm{M}^{+}, 20\right.$ \%). Anal. Calcld for $\mathrm{C}_{22} \mathrm{H}_{19} \mathrm{~N}_{5}$ : C, 74.77; H, 5.42; N, 19.82. Found: C, 74.59; H, 5.51; N, 19.65.

3-p-nitrophenyl-1,5-di-p-tolylformazan (4k). A solution of p-tolylhydrazine hydrochloride (1.60 g, 10 mmol) was combined with triethylamine $(1.4 \mathrm{~mL}, 10 \mathrm{mmol})$ and methanol $(20 \mathrm{~mL})$. After $30 \mathrm{~min}$ of stirring 4-nitrobenzaldehyde $(1.50 \mathrm{~g}, 10 \mathrm{mmol})$ was added and the mixture was stirred for $30 \mathrm{~min}$ at which time an orange ppt formed. The solution was then treated with sodium acetate $(1.75 \mathrm{~g}, 21 \mathrm{mmol})$, sodium hydroxide $(1.25 \mathrm{~g}, 31 \mathrm{mmol})$, and methanol $(80 \mathrm{~mL})$. After $30 \mathrm{~min}$ of stirring a solution of diazonium salt prepared by stirring p-toluidine $(1.07 \mathrm{~g}, 10 \mathrm{mmol})$, sodium nitrite $(0.75 \mathrm{~g}, 11 \mathrm{mmol})$, water $(10 \mathrm{~mL})$, and hydrochloric acid $(5 \mathrm{~mL})$ at $0^{\circ} \mathrm{C}$ for $30 \mathrm{~min}$ was added. The solution immediately turned blood red and after $5 \mathrm{~h}$ was taken to dryness in vacuo. Column chromatography (neutral alumina, dichloromethane) followed by trituration with methanol afforded $\mathbf{4 k}$ as a brick red microcrystalline solid, yield $2.1 \mathrm{~g}(56.3 \%) . \mathrm{Mp} . \quad 216-218^{\circ} \mathrm{C} .{ }^{1} \mathrm{H}$ NMR $\left(\mathrm{CD}_{2} \mathrm{Cl}_{2}\right): \delta 15.81(\mathrm{~s}, 1 \mathrm{H}), 8.31\left(\mathrm{~d}, 2 \mathrm{H},{ }^{3} \mathrm{~J}=9\right.$ $\mathrm{Hz}), 8.25\left(\mathrm{~d}, 2 \mathrm{H},{ }^{3} \mathrm{~J}=9 \mathrm{~Hz}\right), 7.62\left(\mathrm{~d}, 4 \mathrm{H},{ }^{3} \mathrm{~J}=8 \mathrm{~Hz}\right), 7.31\left(\mathrm{~d}, 4 \mathrm{H},{ }^{3} \mathrm{~J}=8 \mathrm{~Hz}\right), 2.42(\mathrm{~s}, 6 \mathrm{H}) \mathrm{ppm} .{ }^{13} \mathrm{C}$ $\operatorname{NMR}\left(\mathrm{CD}_{2} \mathrm{Cl}_{2}\right): \delta 147.3,145.8,144.7,139.3,130.7,126.2,124.3,119.5,21.6$ ppm. FT-IR (KBr): 1592 (s), $1494(\mathrm{~s}), 1334(\mathrm{~s}), 1250(\mathrm{~s}) \mathrm{cm}^{-1}$. UV-Vis $\left(\mathrm{CH}_{2} \mathrm{Cl}_{2}\right): \lambda_{\max } 267 \mathrm{~nm}(\varepsilon=20000), 420 \mathrm{~nm}(\varepsilon=16500)$, $483 \mathrm{~nm}(\varepsilon=20750)$. MS (EI): $m / z 373\left(\mathrm{M}^{+}, 10 \%\right)$. Anal. Calcld for $\mathrm{C}_{21} \mathrm{H}_{19} \mathrm{~N}_{5} \mathrm{O}_{2}: \mathrm{C}, 67.12 ; \mathrm{H}, 5.25 ; \mathrm{N}$, 15.08. Found: C, 70.19; H, 6.16; N, 15.08 .

1,5-di-p-tolyl-3-p-trifluoromethylphenylformazan (4I). Yield 59.9 \%. Mp. $\quad 188-190^{\circ} \mathrm{C} . \quad{ }^{1} \mathrm{H}$ NMR $\left(\mathrm{CD}_{2} \mathrm{Cl}_{2}\right): \delta 15.63(\mathrm{~s}, 1 \mathrm{H}), 8.24\left(\mathrm{~d}, 2 \mathrm{H},{ }^{3} \mathrm{~J}=8 \mathrm{~Hz}\right), 7.67\left(\mathrm{~d}, 2 \mathrm{H},{ }^{3} \mathrm{~J}=8 \mathrm{~Hz}\right), 7.59\left(\mathrm{~d}, 4 \mathrm{H},{ }^{3} \mathrm{~J}=8 \mathrm{~Hz}\right), 7.28$ $\left(\mathrm{d}, 4 \mathrm{H},{ }^{3} \mathrm{~J}=8 \mathrm{~Hz}\right), 2.40(\mathrm{~s}, 6 \mathrm{H}) \mathrm{ppm} .{ }^{13} \mathrm{C} \mathrm{NMR}\left(\mathrm{CD}_{2} \mathrm{Cl}_{2}\right): \delta 146.0,141.9,139.8,138.8,130.6,126.1$ 125.8, 119.3, 21.6 ppm. FT-IR (KBr): $1613(\mathrm{~m}), 1506(\mathrm{~m}), 1497(\mathrm{~m}), 1322(\mathrm{~s}), 1105(\mathrm{~s}), 1067(\mathrm{~s}) \mathrm{cm}^{-1}$. 
UV-Vis $\left(\mathrm{CH}_{2} \mathrm{Cl}_{2}\right): \lambda_{\max } 268 \mathrm{~nm}(\varepsilon=15250), 320 \mathrm{~nm}(\varepsilon=27500), 488 \mathrm{~nm}(\varepsilon=21000) . \mathrm{MS}(\mathrm{EI}): \mathrm{m} / z 396$ $\left(\mathrm{M}^{+}, 10 \%\right)$. Anal. Calcld for $\mathrm{C}_{22} \mathrm{H}_{19} \mathrm{~F}_{3} \mathrm{~N}_{4}: \mathrm{C}, 66.66 ; \mathrm{H}, 4.83 ; \mathrm{N}, 14.13$. Found: C, 66.57; H, 4.82; N, 14.14.

3-p-tolyl-1,5-di-p-trifluoromethylphenylformazan (4m). Yield 44.6 \%. Mp. 164-166 ${ }^{\circ} \mathrm{C} .{ }^{1} \mathrm{H}$ NMR $\left(\mathrm{CD}_{2} \mathrm{Cl}_{2}\right): \delta 14.87(\mathrm{~s}, 1 \mathrm{H}), 7.96\left(\mathrm{~d}, 2 \mathrm{H},{ }^{3} \mathrm{~J}=8 \mathrm{~Hz}\right), 7.76\left(\mathrm{~d}, 4 \mathrm{H},{ }^{3} \mathrm{~J}=9 \mathrm{~Hz}\right), 7.72\left(\mathrm{~d}, 4 \mathrm{H},{ }^{3} \mathrm{~J}=9 \mathrm{~Hz}\right), 7.26$ $\left(\mathrm{d}, 2 \mathrm{H},{ }^{3} \mathrm{~J}=8 \mathrm{~Hz}\right), 2.41(\mathrm{~s}, 3 \mathrm{H}) \mathrm{ppm} .{ }^{13} \mathrm{C} \mathrm{NMR}\left(\mathrm{CD}_{2} \mathrm{Cl}_{2}\right): \delta 150.8,142.9,139.0,134.1,130.8,129.8$, $129.5,129.1,127.3,127.2,126.6,123.4,123.0,119.4,114.6,21.6$ ppm. FT-IR (KBr): 1613 (s), 1511 (m), $1418(\mathrm{~m}), 1322(\mathrm{~s}), 1061(\mathrm{~s}) \mathrm{cm}^{-1}$. UV-Vis $\left(\mathrm{CH}_{2} \mathrm{Cl}_{2}\right): \lambda_{\max } 273 \mathrm{~nm}(\varepsilon=21500), 297 \mathrm{~nm}(\varepsilon=$ 27500), $497 \mathrm{~nm}(\varepsilon=14500)$. MS (EI): $m / z 450\left(\mathrm{M}^{+}, 10 \%\right)$. Anal. Calcld for $\mathrm{C}_{22} \mathrm{H}_{16} \mathrm{~F}_{6} \mathrm{~N}_{4}: \mathrm{C}, 58.67 ; \mathrm{H}$, 3.58; N, 12.44. Found: C, 58.73; H, 3.37; N, 12.41 .

General procedure for verdazyls 1a-e, g-i: 1,3,5-triphenylverdazyl (1a). In a typical procedure 4a (0.46 g, $1.5 \mathrm{mmol})$ was combined with formaldehyde solution $(1.7 \mathrm{~mL}, 23 \mathrm{mmol}), 2 \mathrm{M}$ sodium hydroxide (3.5 mL, $7 \mathrm{mmol})$, and dimethylformamide $(25 \mathrm{~mL})$ and allowed to stir in air for 3 hours at which time the color of the solution had changed from red to green. The mixture was then treated with diethyl ether $(100 \mathrm{~mL})$ and the organic layer washed with water $(5 \mathrm{x} 100 \mathrm{~mL})$ to remove dimethylformamide, followed by removal of the ether in vacuo. Recrystallization from a saturated methanolic solution afforded $\mathbf{1 a}$ as a green microcrystalline solid, yield $0.26 \mathrm{~g}(54.2 \%)$. Mp. 128$130^{\circ} \mathrm{C}$. FT-IR (KBr): 1586 (s), $1490(\mathrm{~s}), 1264(\mathrm{~m}), 1145(\mathrm{~m}), 752(\mathrm{~s}) \mathrm{cm}^{-1}$. UV-Vis $\left(\mathrm{CH}_{2} \mathrm{Cl}_{2}\right): \lambda_{\max } 273$ $\mathrm{nm}(\varepsilon=62250), 405 \mathrm{~nm}(\varepsilon=7250), 716 \mathrm{~nm}(\varepsilon=3500)$. MS (EI): $m / z 313\left(\mathrm{M}^{+}, 70 \%\right)$. Anal. Calcld for $\mathrm{C}_{20} \mathrm{H}_{17} \mathrm{~N}_{4}: \mathrm{C}, 76.65 ; \mathrm{H}, 5.47 ; \mathrm{N}, 17.88$. Found: C, 76.25; H, 5.10; N, 17.37.

1,5-di-p-methoxyphenyl-3-p-tolylverdazyl (1b). Yield 75.6 \%. Mp. 120-122 ${ }^{\circ} \mathrm{C}$. FT-IR (KBr): 1504 $(\mathrm{s}), 1248(\mathrm{~s}), 1033(\mathrm{~m}), 821(\mathrm{~m}) \mathrm{cm}^{-1}$. UV-Vis $\left(\mathrm{CH}_{2} \mathrm{Cl}_{2}\right): \lambda_{\max } 287 \mathrm{~nm}(\varepsilon=27500), 327 \mathrm{~nm}(\varepsilon=11750)$, $414 \mathrm{~nm}(\varepsilon=8250), 736 \mathrm{~nm}(\varepsilon=4250)$. MS (EI): $m / z 387\left(\mathrm{M}^{+}, 100 \%\right)$. Anal. Calcld for $\mathrm{C}_{23} \mathrm{H}_{23} \mathrm{~N}_{4} \mathrm{O}_{2}$ : C, 71.30; H, 5.98; N, 14.46. Found: C, 71.23; H, 5.97; N, 14.20. 
1,3,5-tri-p-tolylverdazyl (1c). Yield 57.7\%. Mp. 136-138C. FT-IR (KBr): 1508 (s), 1397 (m), 1260

(m), $1143(\mathrm{~s}), 825(\mathrm{~m}), 798(\mathrm{~m}) \mathrm{cm}^{-1}$. UV-Vis $\left(\mathrm{CH}_{2} \mathrm{Cl}_{2}\right): \lambda_{\max } 248 \mathrm{~nm}(\varepsilon=12500), 284 \mathrm{~nm}(\varepsilon=22000)$, $325 \mathrm{~nm}(\varepsilon=10000), 403 \mathrm{~nm}(\varepsilon=6750), 727 \mathrm{~nm}(\varepsilon=3250) . \quad \mathrm{MS}(\mathrm{EI}): m / z 354\left(\mathrm{M}-1^{+}, 100 \%\right)$. Anal. Calcld for $\mathrm{C}_{23} \mathrm{H}_{23} \mathrm{~N}_{4}$ : C, 77.72; H, 6.52; N, 15.76. Found: C, 77.13; H, 6.69; N, 15.12 .

1,5-diphenyl-3-p-tolylverdazyl (1d). Yield 27.2\%. Mp. 138-140C. FT-IR (KBr): 1590 (s), 1494 (s), $1397(\mathrm{~m}), 1143(\mathrm{~s}), 746(\mathrm{~s}) \mathrm{cm}^{-1}$. UV-Vis $\left(\mathrm{CH}_{2} \mathrm{Cl}_{2}\right): \lambda_{\max } 246 \mathrm{~nm}(\varepsilon=10000), 280 \mathrm{~nm}(\varepsilon=23000), 320$ $\mathrm{nm}(\varepsilon=12000), 398 \mathrm{~nm}(\varepsilon=7500), 721 \mathrm{~nm}(\varepsilon=3500)$. MS (EI): $m / z 327\left(\mathrm{M}^{+}, 80 \%\right)$. Anal. Calcld for $\mathrm{C}_{21} \mathrm{H}_{19} \mathrm{~N}_{4}$ : C, 77.04; H, 5.85; N, 17.11. Found: C, 76.43; H, 5.57; N, 16.64.

1,5-di-p-chlorophenyl-3-p-tolylverdazyl (1e). Yield 83.7\%. Mp. 122-124 C. FT-IR (KBr): 1584 (m), $1488(\mathrm{~s}), 1091(\mathrm{~s}), 818(\mathrm{~s}) \mathrm{cm}^{-1}$. UV-Vis $\left(\mathrm{CH}_{2} \mathrm{Cl}_{2}\right): \lambda_{\max } 283 \mathrm{~nm}(\varepsilon=25000), 327 \mathrm{~nm}(\varepsilon=13250)$, $395 \mathrm{~nm}(\varepsilon=9000), 730 \mathrm{~nm}(\varepsilon=4050) . \mathrm{MS}(\mathrm{EI}): \mathrm{m} / z 396\left(\mathrm{M}^{+}, 40 \%\right)$. Anal. Calcld for $\mathrm{C}_{21} \mathrm{H}_{17} \mathrm{Cl}_{2} \mathrm{~N}_{4}: \mathrm{C}_{\text {, }}$ 63.65; H, 4.32; N, 14.14. Found: C, 63.73; H, 4.41; N, 14.17.

3-p-methoxyphenyl-1,5-di-p-tolylverdazyl (1g). Yield $74.3 \%$. Mp. $56-58^{\circ} \mathrm{C}(\mathrm{dec}) . \quad \mathrm{FT}-\mathrm{IR}(\mathrm{KBr})$ : $1709(\mathrm{~m}), 1610(\mathrm{~m}), 1507(\mathrm{~s}), 1249(\mathrm{~s}) \mathrm{cm}^{-1}$. UV-Vis $\left(\mathrm{CH}_{2} \mathrm{Cl}_{2}\right): \lambda_{\max } 283 \mathrm{~nm}(\varepsilon=26000), 323 \mathrm{~nm}(\varepsilon=$

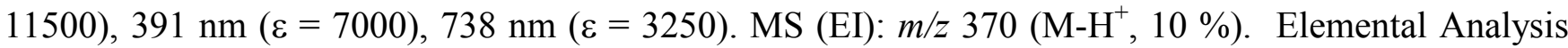
could not be obtained as this compound decomposes over a period of several hours.

3-phenyl-1,5-di-p-tolylverdazyl (1h). . Yield 50.2 \%. Mp. 122-124 C. FT-IR (KBr): 1599 (m), 1504 (s), $1262(\mathrm{~s}), 1143(\mathrm{~s}) \mathrm{cm}^{-1}$. UV-Vis $\left(\mathrm{CH}_{2} \mathrm{Cl}_{2}\right): \lambda_{\max } 247 \mathrm{~nm}(\varepsilon=13750), 286 \mathrm{~nm}(\varepsilon=24000), 323 \mathrm{~nm}$ $(\varepsilon=13000), 410 \mathrm{~nm}(\varepsilon=8500), 725 \mathrm{~nm}(\varepsilon=4500)$. MS (EI): $m / z 341\left(\mathrm{M}^{+}, 65 \%\right)$. Anal. Calcld for $\mathrm{C}_{22} \mathrm{H}_{21} \mathrm{~N}_{4}$ : C, 77.39; H, 6.20; N, 16.41. Found: C, 77.49; H, 6.43; N, 16.60.

3-p-chlorophenyl-1,5-di-p-tolylverdazyl (1i). Yield 68.1 \%. Mp. 117-119². FT-IR (KBr): 1599 (s), $1563(\mathrm{~s}), 1509$ (s), $1088(\mathrm{~s}) \mathrm{cm}^{-1}$. UV-Vis $\left(\mathrm{CH}_{2} \mathrm{Cl}_{2}\right): \lambda_{\max } 256 \mathrm{~nm}(\varepsilon=28000), 438 \mathrm{~nm}(\varepsilon=2500), 740$

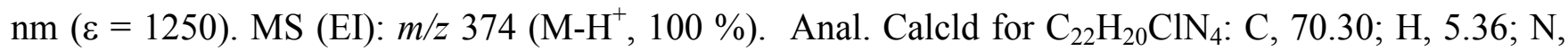
14.91. Found: C, 69.93; H, 5.10; N, 14.94. 
1,3,5-triphenyl-6-oxoverdazyl (2a). 1,3,5-triphenyl-1,2,4,5-tetrazane-6-oxide ${ }^{12}(0.600 \mathrm{~g}, 1.8 \mathrm{mmol})$, silver carbonate $(0.750 \mathrm{~g}, 2.7 \mathrm{mmol})$, and celite $(0.500 \mathrm{~g})$ were combined in methanol $(25 \mathrm{~mL})$ and allowed to stir for 15 hours. The celite, now plated with silver metal, was filtered off and the solvent removed in vacuo to afford a dark red powder. Recrystallization from a saturated methanolic solution afforded $2 \mathbf{a}$ as red needles, yield $0.420 \mathrm{~g}(71.3 \%)$. Mp. 206-208 ${ }^{\circ} \mathrm{C}$. FT-IR (KBr): $1695(\mathrm{~s}) \mathrm{cm}^{-1}$. UVVis $\left(\mathrm{CH}_{2} \mathrm{Cl}_{2}\right): \lambda_{\max } 255 \mathrm{~nm}(\varepsilon=21250), 291 \mathrm{~nm}(\varepsilon=8000), 320 \mathrm{~nm}(\varepsilon=12750), 422 \mathrm{~nm}(\varepsilon=1250)$, $537 \mathrm{~nm}(\varepsilon=2500), 561 \mathrm{~nm}(\varepsilon=2500)$. MS (EI): $m / z 328\left(\mathrm{M}+\mathrm{H}^{+}, 60 \%\right)$. Anal. Calcd. for $\mathrm{C}_{20} \mathrm{H}_{15} \mathrm{~N}_{4} \mathrm{O}$ : C, 73.38; H, 4.62; N, 17.11. Found: C, 73.28; H, 4.54; N, 17.26.

1,5-diisopropyl-3-(2'-methylimidazol-5'-yl)-1,2,4,5-tetrazane-6-oxide (8). Carbonic acid bis(1isopropylhydrazide) $\cdot 2 \mathrm{HCl}^{11}(1.66 \mathrm{~g}, 6.7 \mathrm{mmol})$, sodium acetate $(1.289 \mathrm{~g}, 13.4 \mathrm{mmol})$, and 2methylimidazolecarboxaldehyde $(0.74 \mathrm{~g}, 6.7 \mathrm{mmol})$ were combined in ethanol $(200 \mathrm{~mL})$ and stirred overnight. The solvent was removed under reduced pressure and the crude product recrystallized from heptane to give a white crystalline solid, yield $0.892 \mathrm{~g}(50 \%)$. Mp. $192-193^{\circ} \mathrm{C} .{ }^{1} \mathrm{H} \mathrm{NMR}\left(\mathrm{CDCl}_{3}\right): \delta$ $6.96(\mathrm{~s}, 1 \mathrm{H}), 6.89(\mathrm{~d}, 1 \mathrm{H}, J=1 \mathrm{~Hz}), 4.65$ (septet, 2H, $J=7 \mathrm{~Hz}), 4.53(\mathrm{t}, 1 \mathrm{H}, J=12 \mathrm{~Hz}), 4.39(\mathrm{~d}, 2 \mathrm{H}, J=$ $12 \mathrm{~Hz}), 3.71(\mathrm{~s}, 3 \mathrm{H}),, 1.08(\mathrm{~d}, 6 \mathrm{H}, J=7 \mathrm{~Hz}), 1.06(\mathrm{~d}, 6 \mathrm{H}, J=7 \mathrm{~Hz}) \mathrm{ppm} .{ }^{13} \mathrm{C} \mathrm{NMR}\left(\mathrm{CDCl}_{3}\right): \delta 153.6$, 142.5, 128.0, 122.3, 64.6, 47.7, 32.9, 19.6, 18.8 ppm. FT-IR (KBr): (br, w), 3220 (m), 2983 (m), 1615 (s), 1495 (m), 1459 (m), $1416(\mathrm{~s}), 1124$ (m), 1067 (m), 922 (m), cm-1. MS (EI): m/z $266\left(\mathrm{M}^{+}, 21 \%\right)$. Anal. Calcd. for $\mathrm{C}_{12} \mathrm{H}_{22} \mathrm{~N}_{6} \mathrm{O}$ : C, 54.11; H, 8.33; N, 31.55. Found: C, 54.02; H, 8.38; N, 31.55.

1,5-diisopropyl-3-(2'-methylimidazol-5'-yl)-6-oxoverdazyl (2f). $p$-benzoquinone (0.35 g, $3.2 \mathrm{mmol})$ was added to a solution of $8(0.57 \mathrm{~g}, 2.1 \mathrm{mmol})$ in toluene $(50 \mathrm{~mL})$ and the mixture was refluxed for 1.5 h. Solvent was removed under reduced pressure to give an oily red product that was purified by flash chromatography $\left(\mathrm{CH}_{2} \mathrm{Cl}_{2}\right.$, alumina) to give the radical as a red solid, yield $0.46 \mathrm{~g}(83 \%)$. Mp. 112 $114^{\circ} \mathrm{C}$. FT-IR (KBr): 1689 (s), 1467 (m), 1242 (m), 1166 (m), $1143(\mathrm{~m}), 1129,778(\mathrm{~m}), \mathrm{cm}^{-1}$. UV-Vis $\left(\mathrm{CH}_{2} \mathrm{Cl}_{2}\right): \lambda_{\max } 261 \mathrm{~nm}(\varepsilon=18000), 412 \mathrm{~nm}(\varepsilon=1200)$. MS (EI): $m / z 263\left(\mathrm{M}^{+}, 5 \%\right)$. Anal. Calcd. for $\mathrm{C}_{12} \mathrm{H}_{19} \mathrm{~N}_{6} \mathrm{O}$ : C, 54.74; H, 7.27; N, 31.92. Found: C, 54.80; H, 7.55; N, 31.89. 


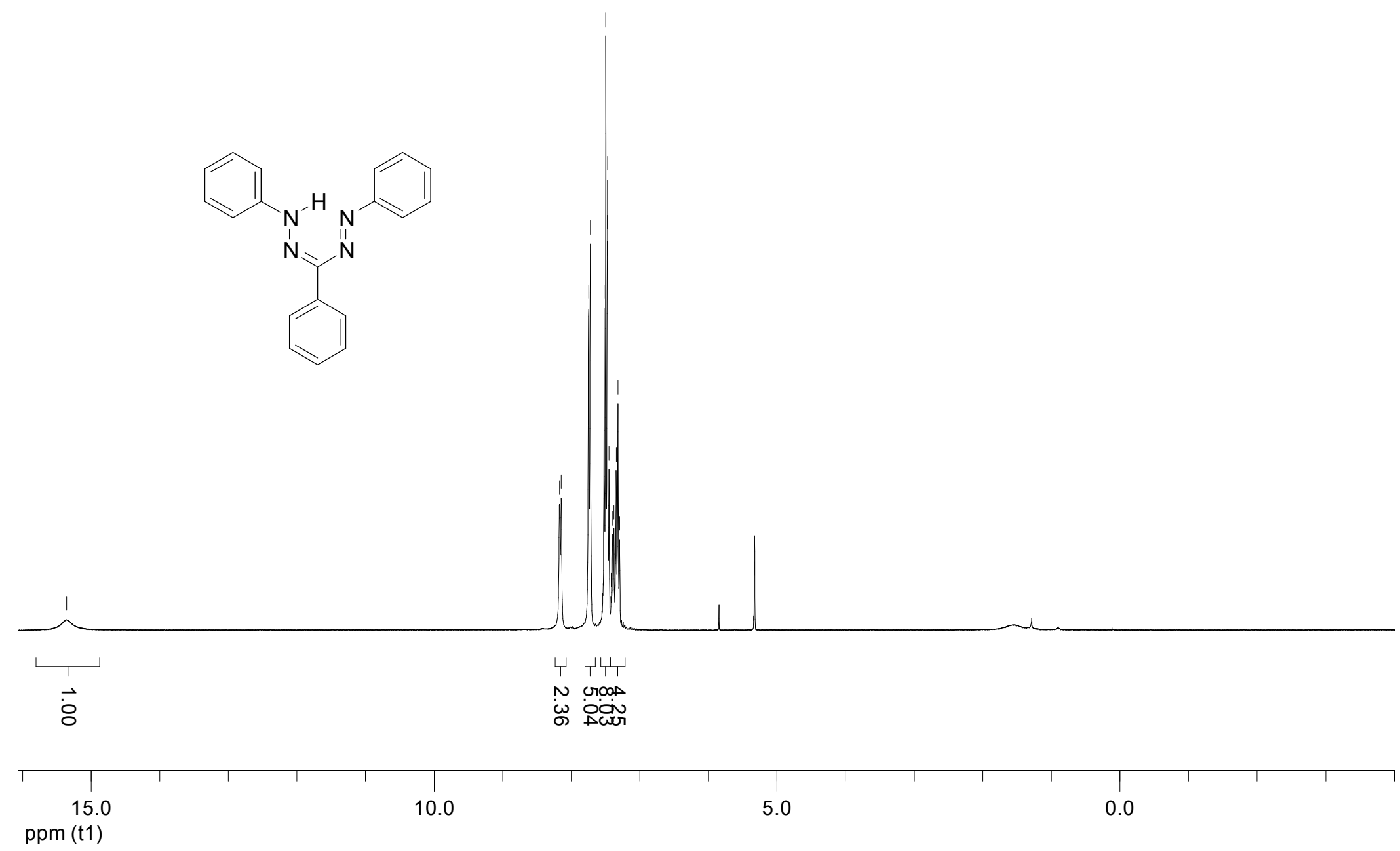

Figure S1. ${ }^{1} \mathrm{H} \mathrm{NMR}$ of $4 \mathbf{a}\left(\mathrm{CD}_{2} \mathrm{Cl}_{2}\right)$ 

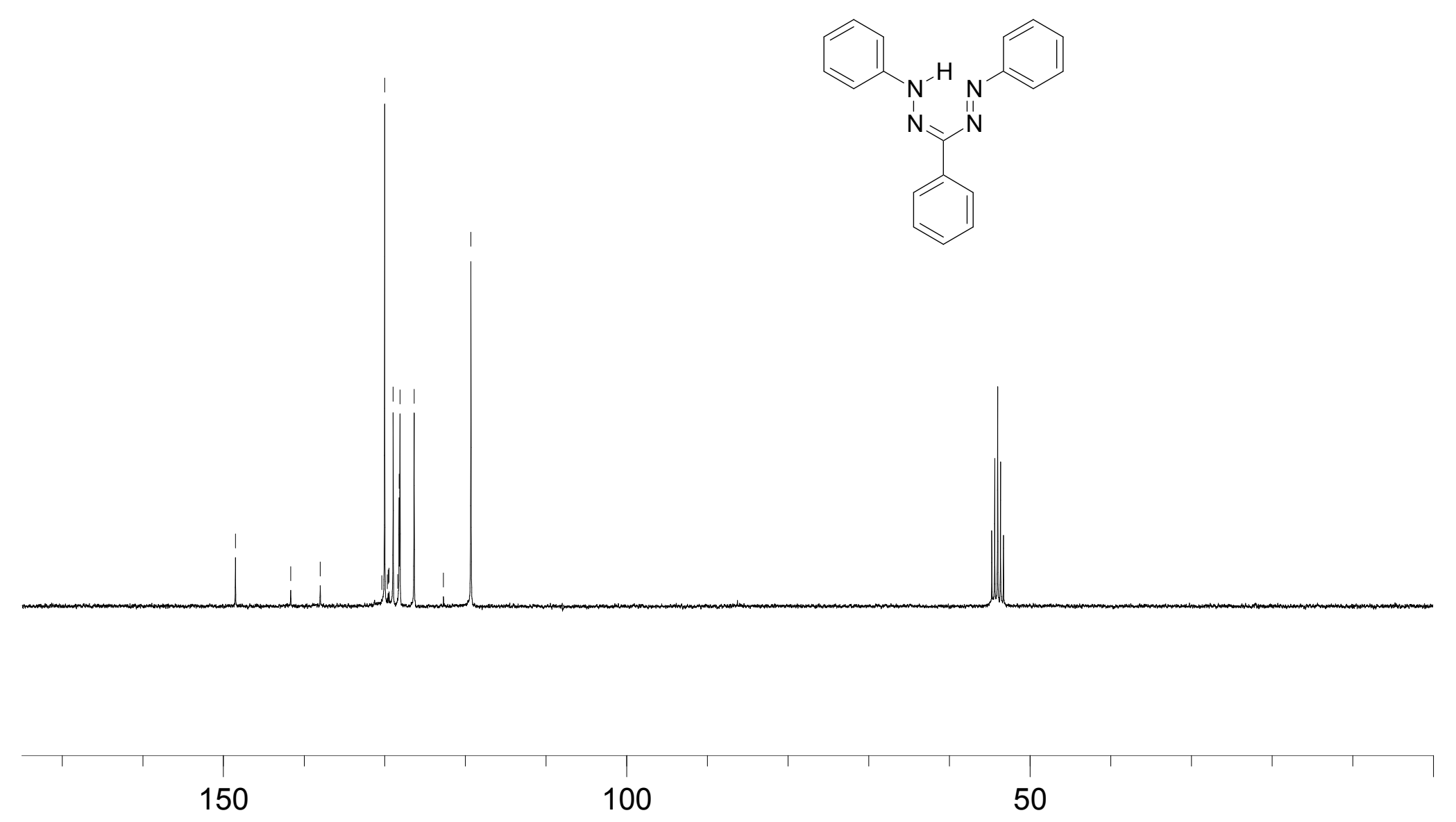

Figure S2. ${ }^{13} \mathrm{C}$ NMR of $4 \mathbf{a}\left(\mathrm{CD}_{2} \mathrm{Cl}_{2}\right)$ 


$$
\begin{aligned}
& \text { 요 } \\
& \text { ñ } \\
& \text { ம }
\end{aligned}
$$
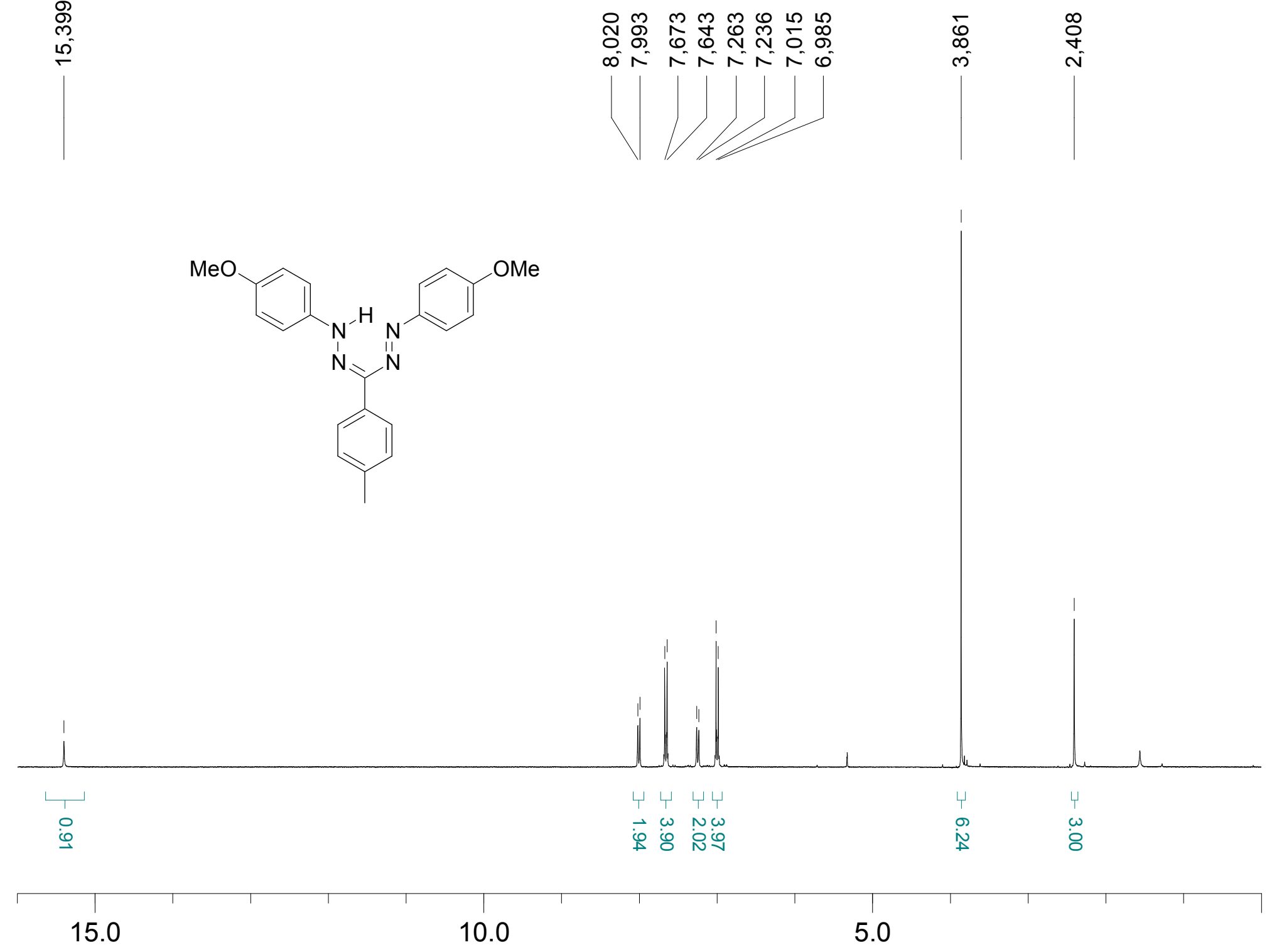

Figure S3. ${ }^{1} \mathrm{H} \mathrm{NMR}$ of $\mathbf{4 b}\left(\mathrm{CD}_{2} \mathrm{Cl}_{2}\right)$ 

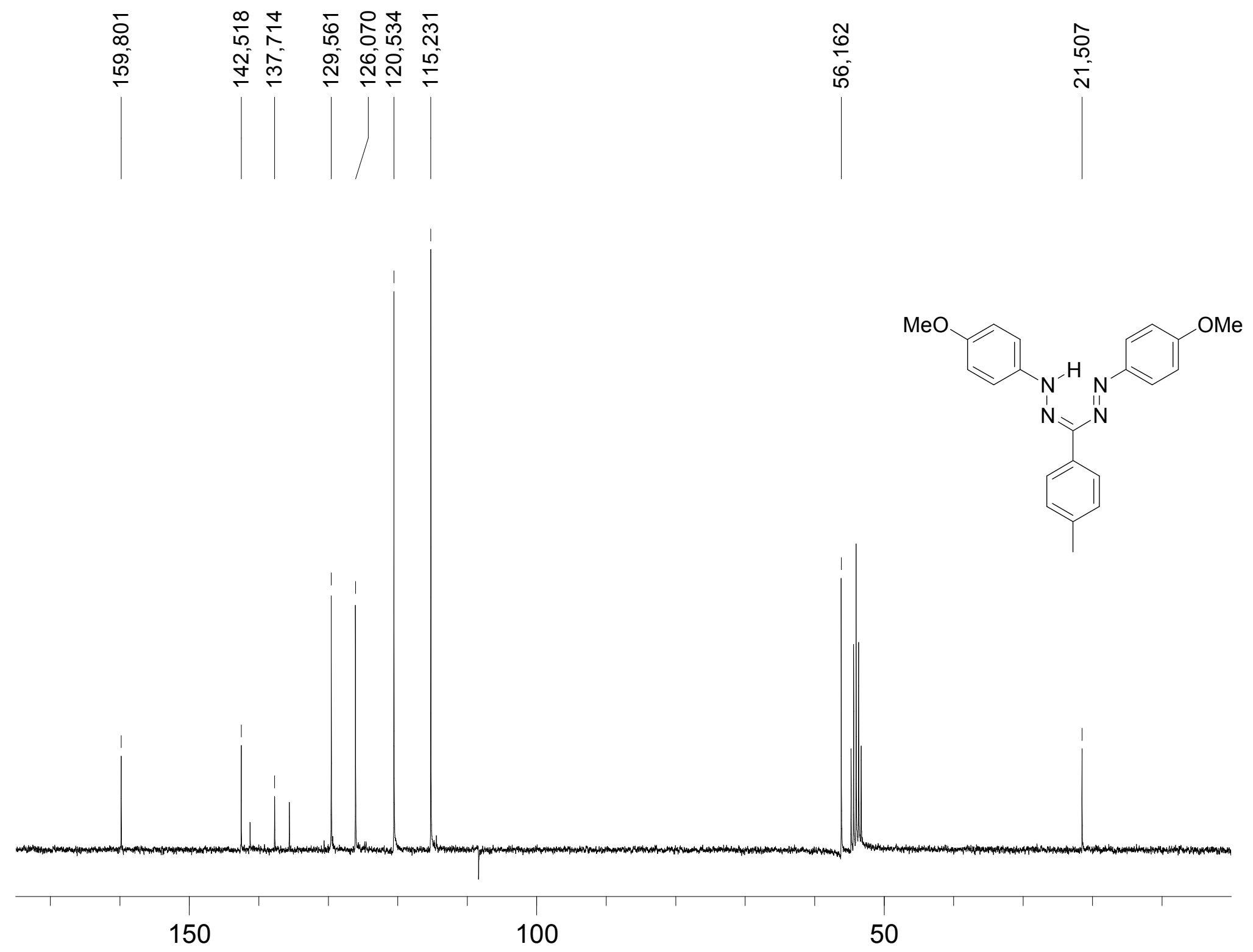

Figure S4. ${ }^{13} \mathrm{C}$ NMR of $\mathbf{4 b}\left(\mathrm{CD}_{2} \mathrm{Cl}_{2}\right)$ 


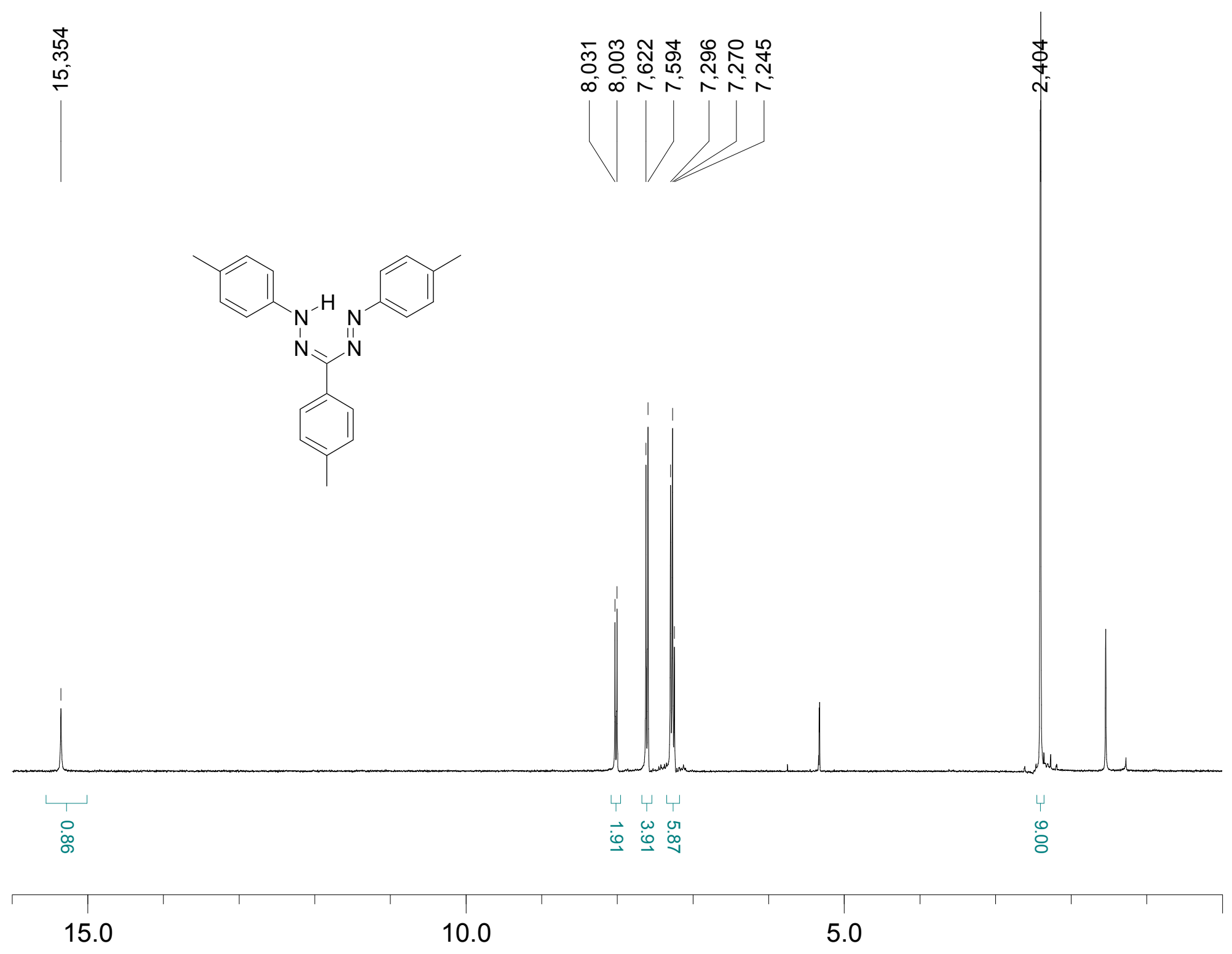

Figure S5. ${ }^{1} \mathrm{H} \mathrm{NMR}$ of $4 \mathbf{c}\left(\mathrm{CD}_{2} \mathrm{Cl}_{2}\right)$ 


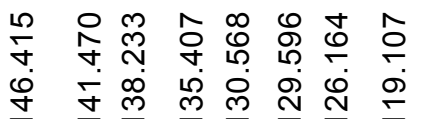

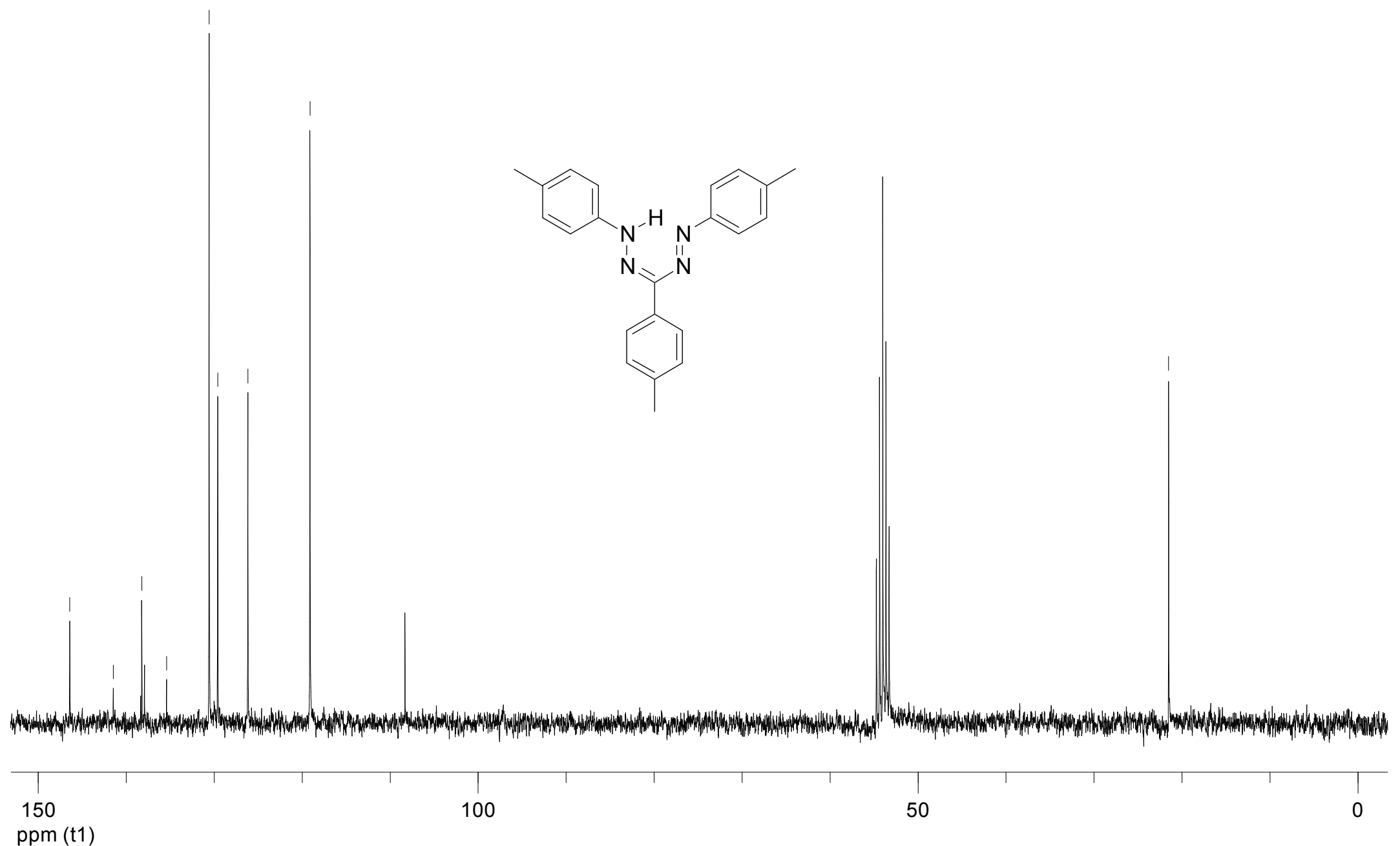

ppm (t1)

Figure S6. ${ }^{13} \mathrm{C}$ NMR of $4 \mathbf{c}\left(\mathrm{CD}_{2} \mathrm{Cl}_{2}\right)$ 


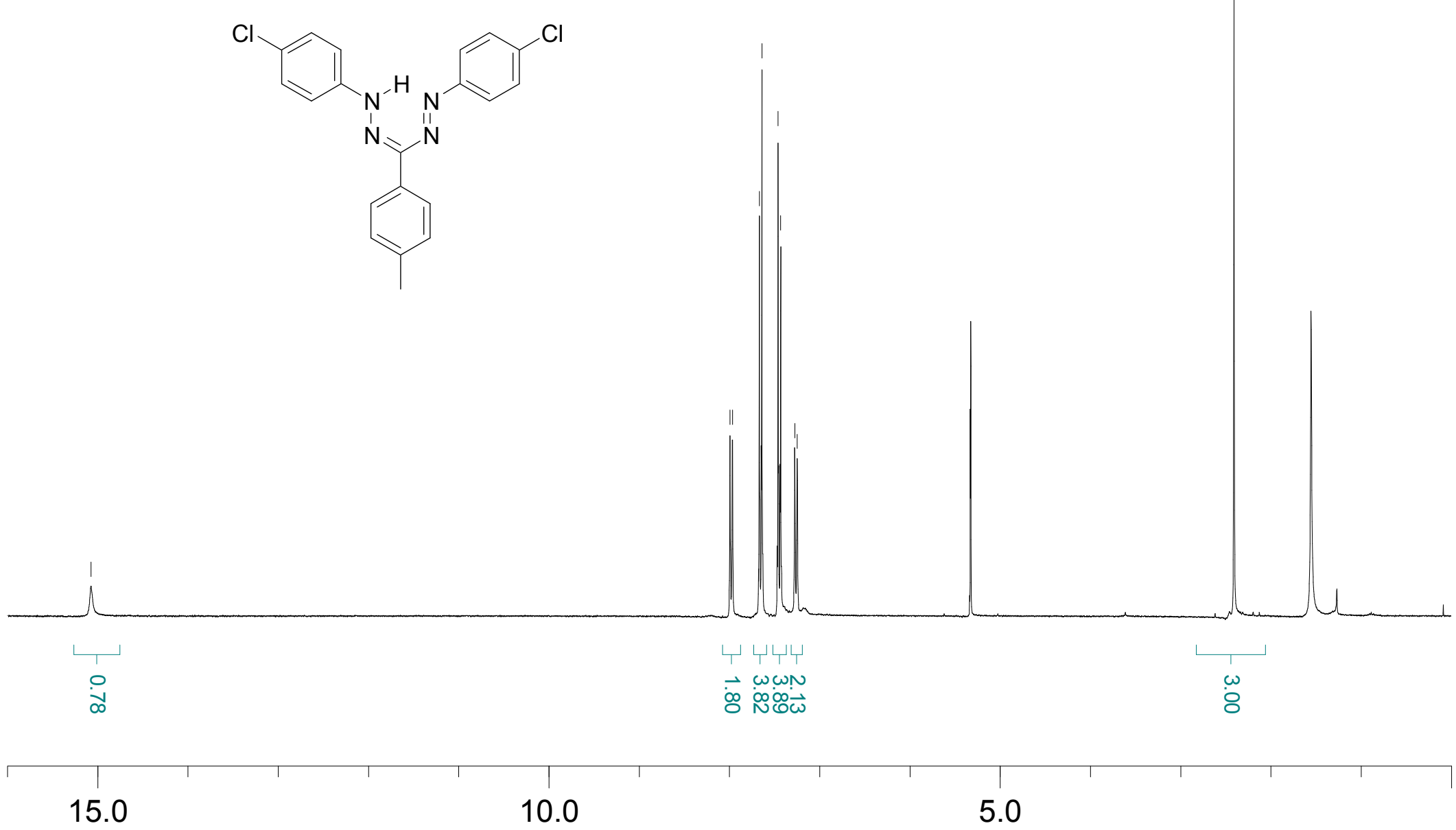

Figure S7. ${ }^{1} \mathrm{H}$ NMR of $4 e\left(\mathrm{CD}_{2} \mathrm{Cl}_{2}\right)$ 


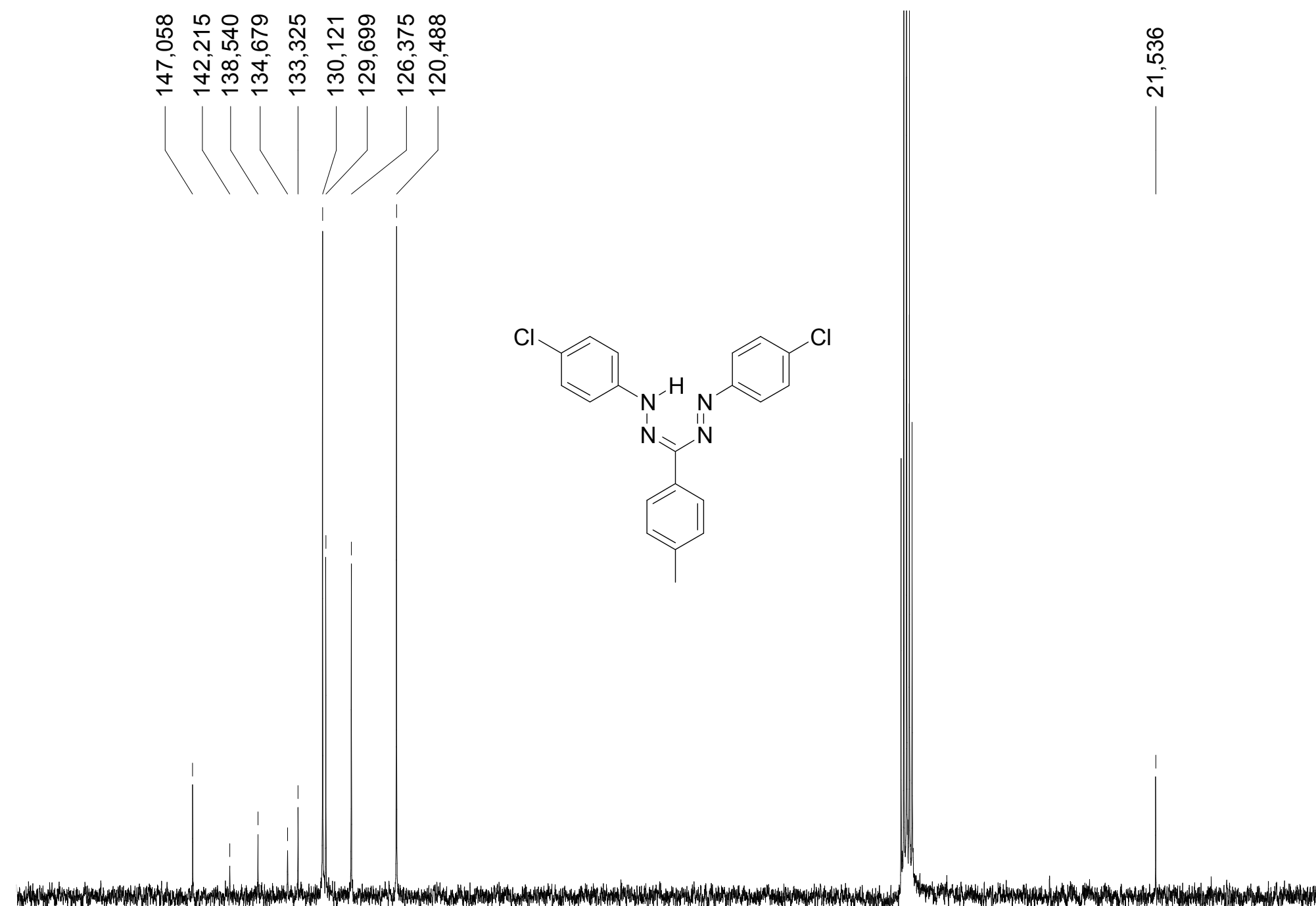

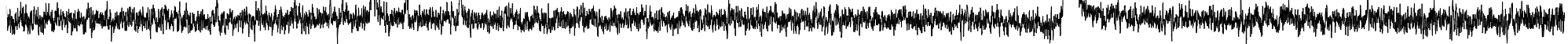

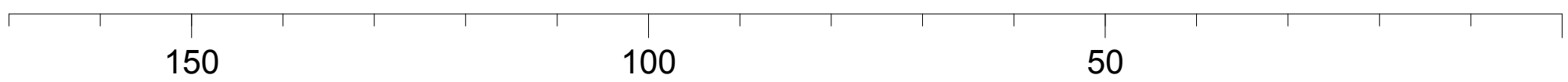

Figure S8. ${ }^{13} \mathrm{C} \mathrm{NMR}$ of $4 \mathrm{e}\left(\mathrm{CD}_{2} \mathrm{Cl}_{2}\right)$ 


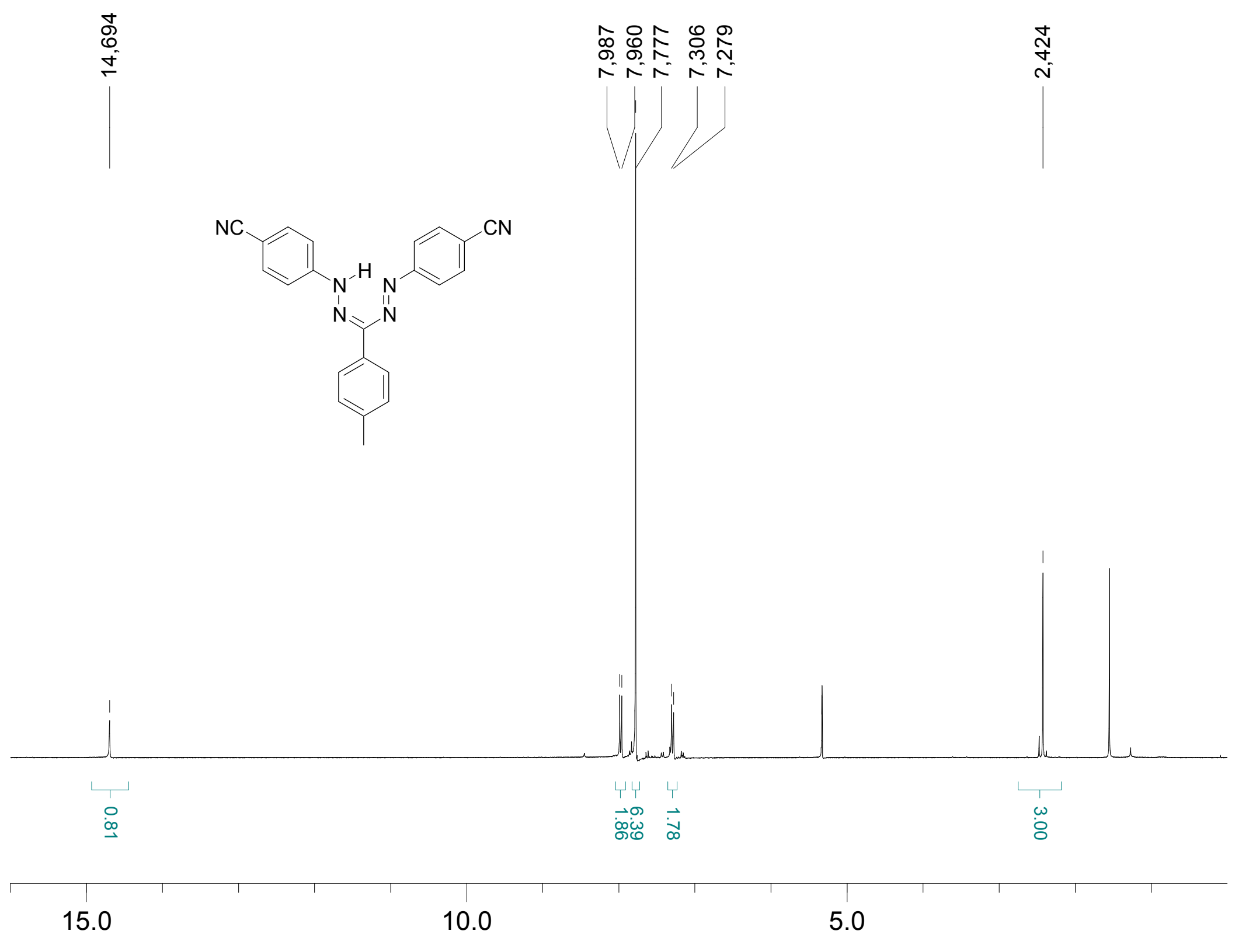

Figure S9. ${ }^{1} \mathrm{H} \mathrm{NMR}$ of $\mathbf{4 f}\left(\mathrm{CD}_{2} \mathrm{Cl}_{2}\right)$ 


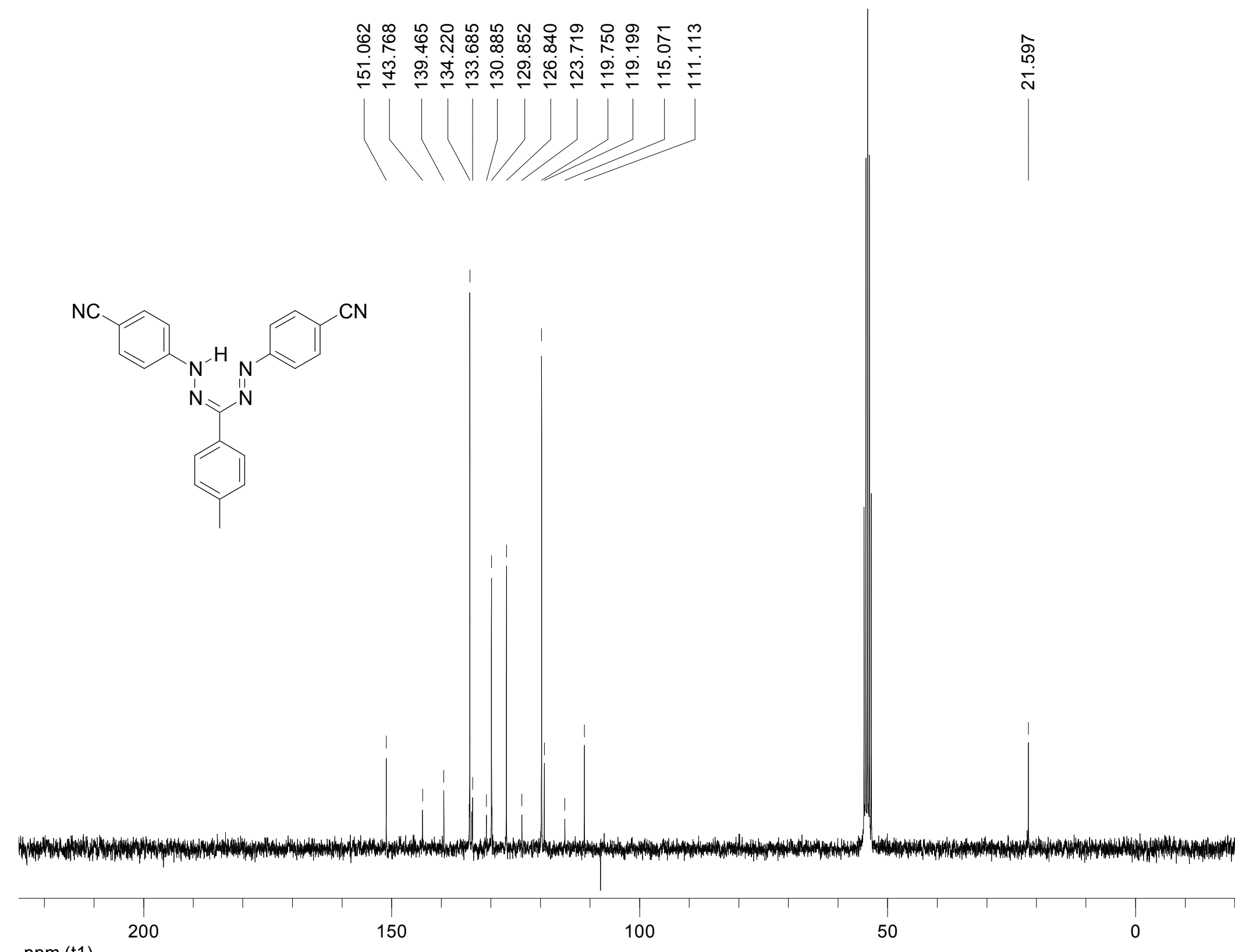

ppm (t1)

Figure S10. ${ }^{13} \mathrm{C}$ NMR of $\mathbf{4 f}\left(\mathrm{CD}_{2} \mathrm{Cl}_{2}\right)$ 


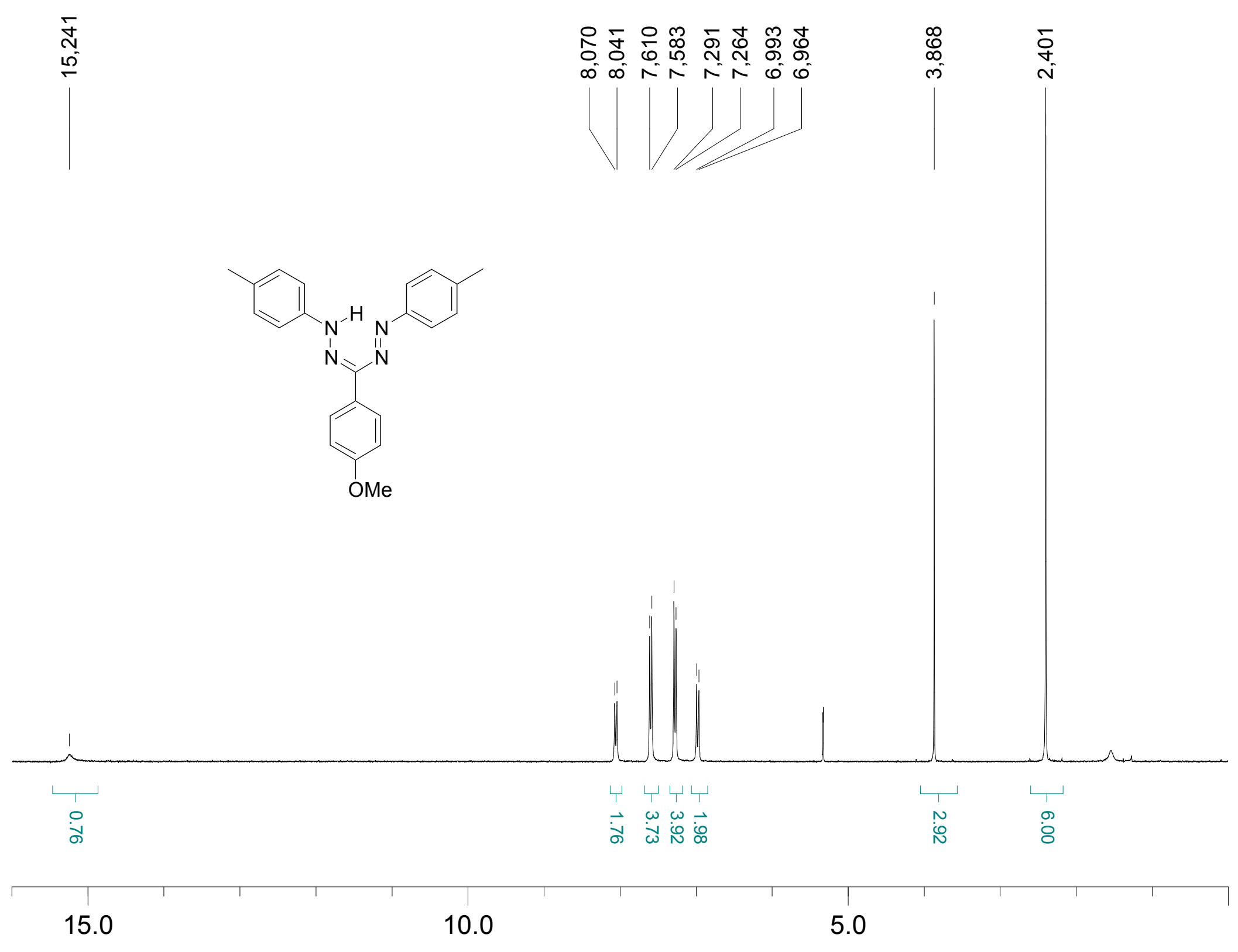

Figure S11. ${ }^{1} \mathrm{H} \mathrm{NMR}$ of $4 \mathrm{~g}\left(\mathrm{CD}_{2} \mathrm{Cl}_{2}\right)$ 

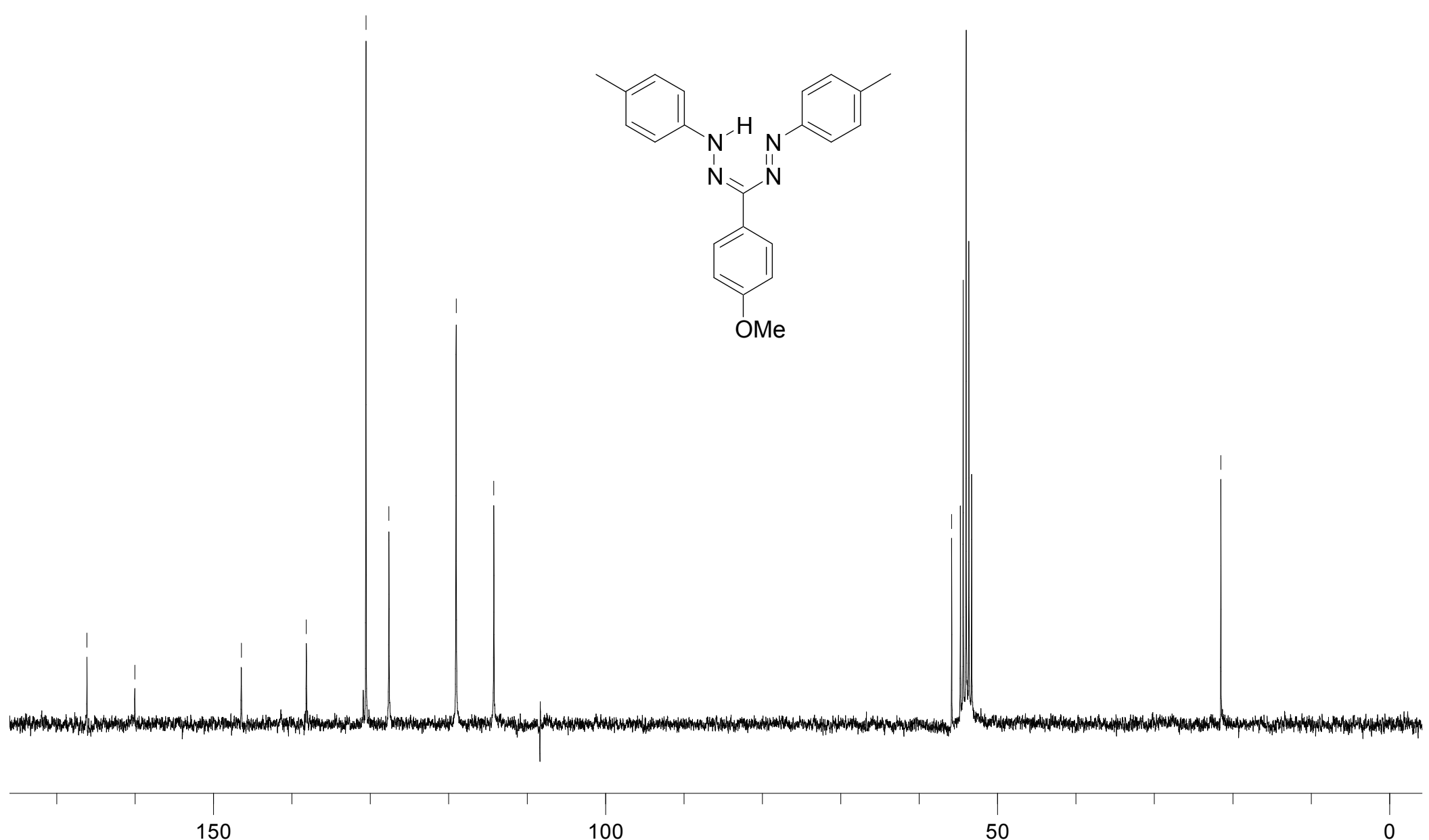

ppm (t1)

Figure S12. ${ }^{13} \mathrm{C}$ NMR of $\mathbf{4 g}\left(\mathrm{CD}_{2} \mathrm{Cl}_{2}\right)$ 

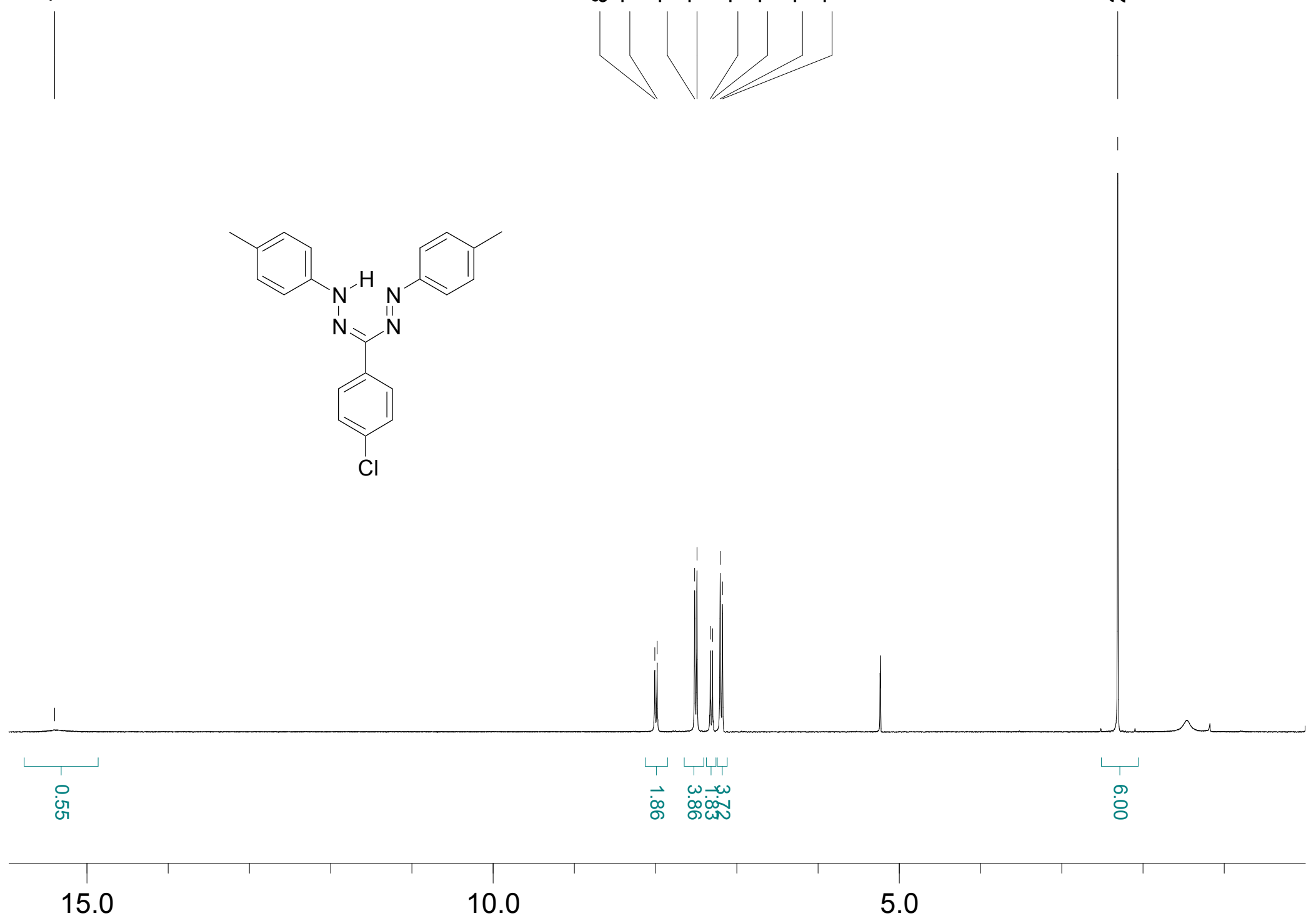

Figure S13. ${ }^{1} \mathrm{H}$ NMR of $4 \mathbf{i}\left(\mathrm{CD}_{2} \mathrm{Cl}_{2}\right)$ 


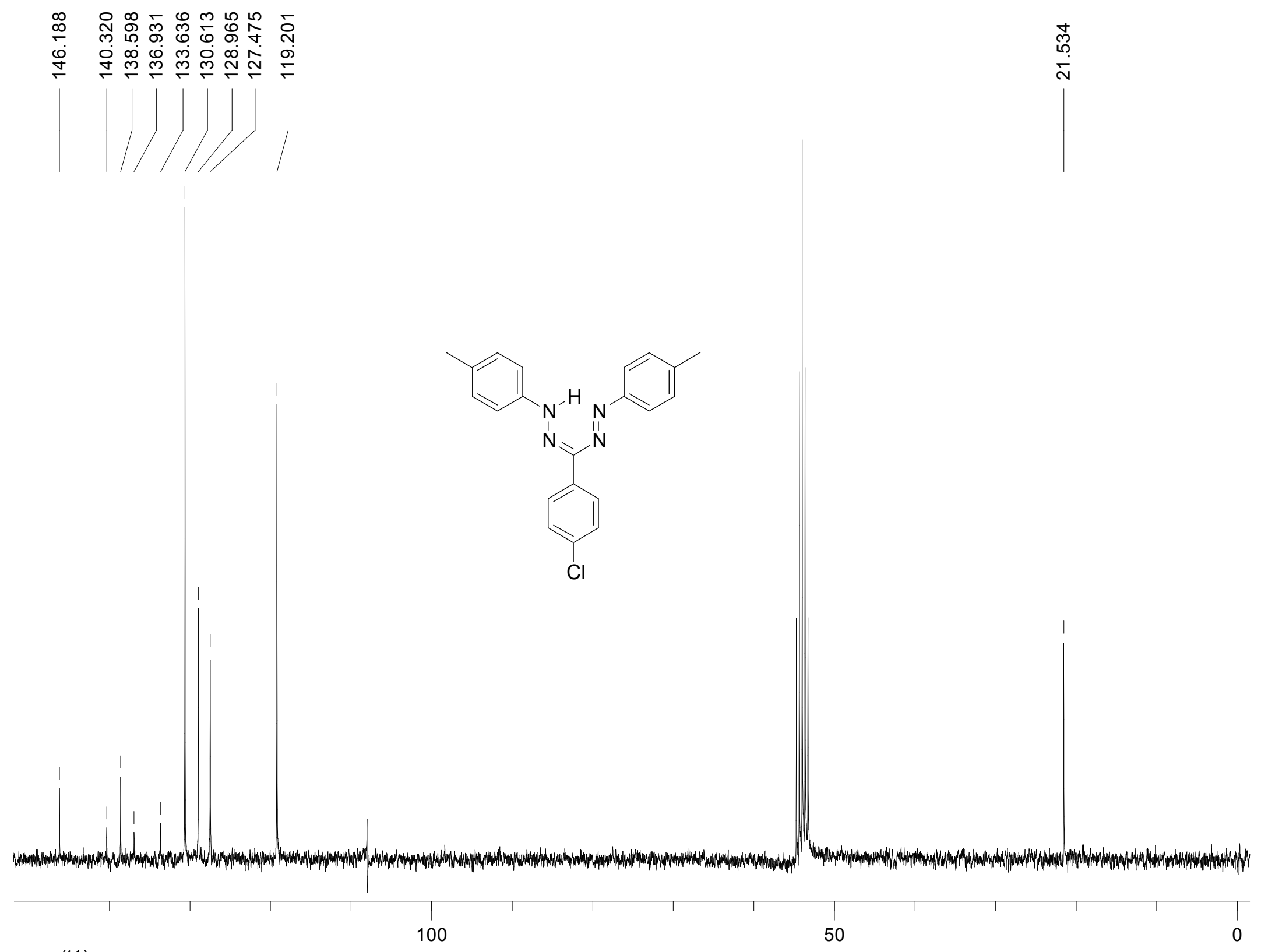

ppm (t1)

Figure S14. ${ }^{13} \mathrm{C}$ NMR of $4 \mathrm{i}\left(\mathrm{CD}_{2} \mathrm{Cl}_{2}\right)$ 


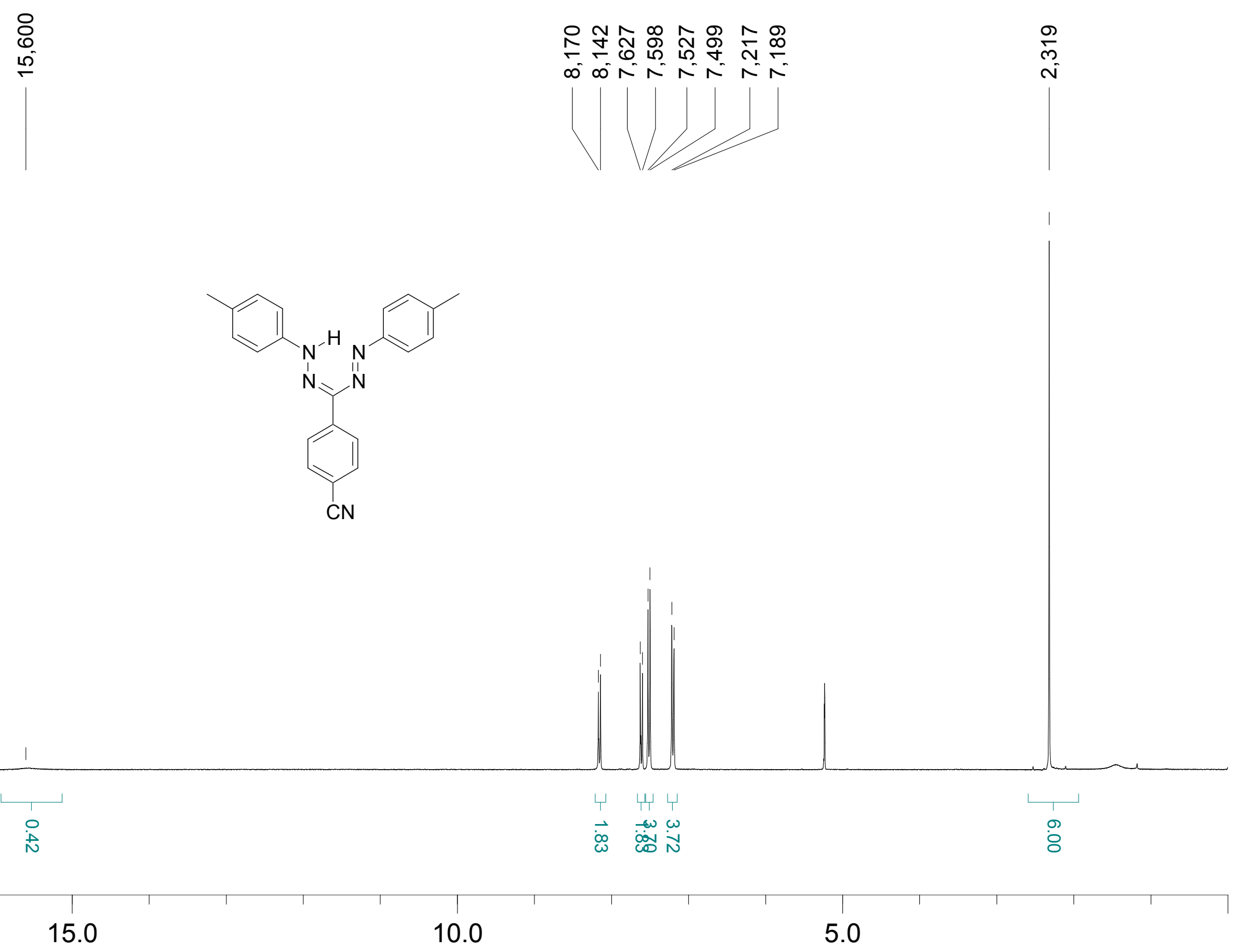

Figure $\mathbf{S 1 5} .{ }^{1} \mathrm{H} \mathrm{NMR}$ of $\mathbf{4 j}\left(\mathrm{CD}_{2} \mathrm{Cl}_{2}\right)$ 

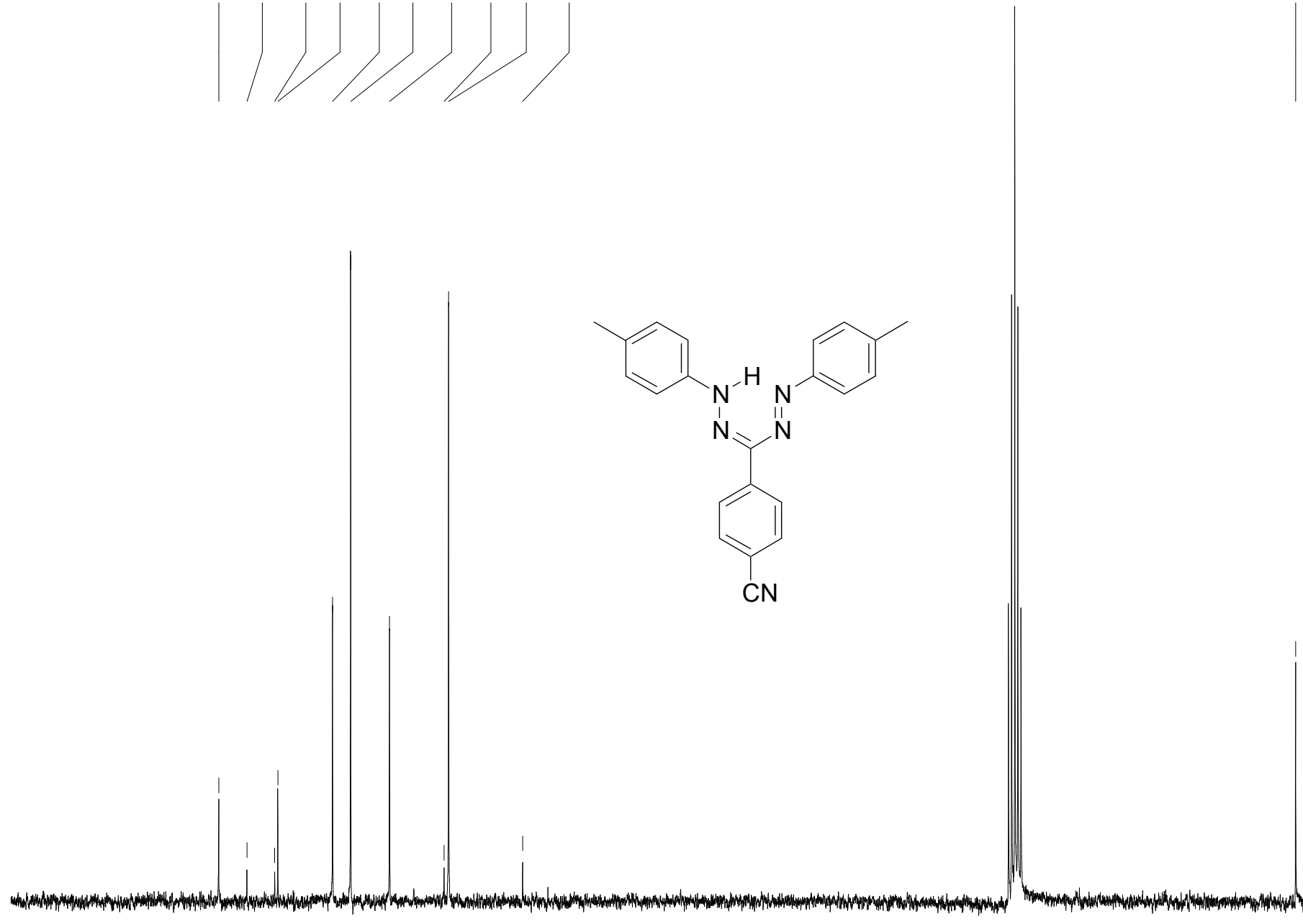

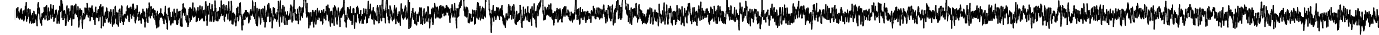

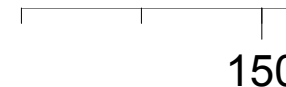

Figure S16. ${ }^{13} \mathrm{C}$ NMR of $4 \mathbf{j}\left(\mathrm{CD}_{2} \mathrm{Cl}_{2}\right)$ 


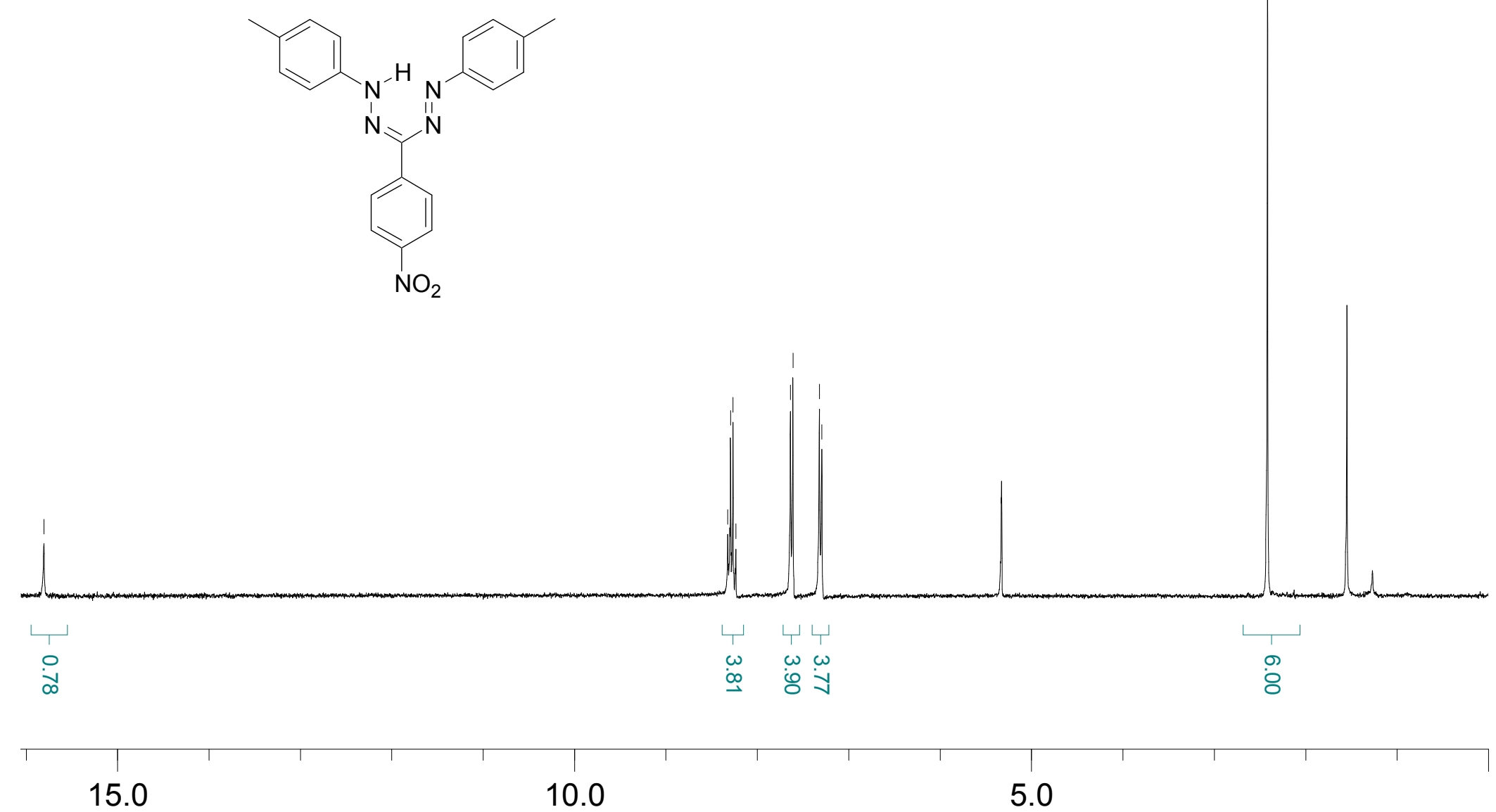

Figure S17. ${ }^{1} \mathrm{H} \mathrm{NMR}$ of $4 \mathbf{k}\left(\mathrm{CD}_{2} \mathrm{Cl}_{2}\right)$ 

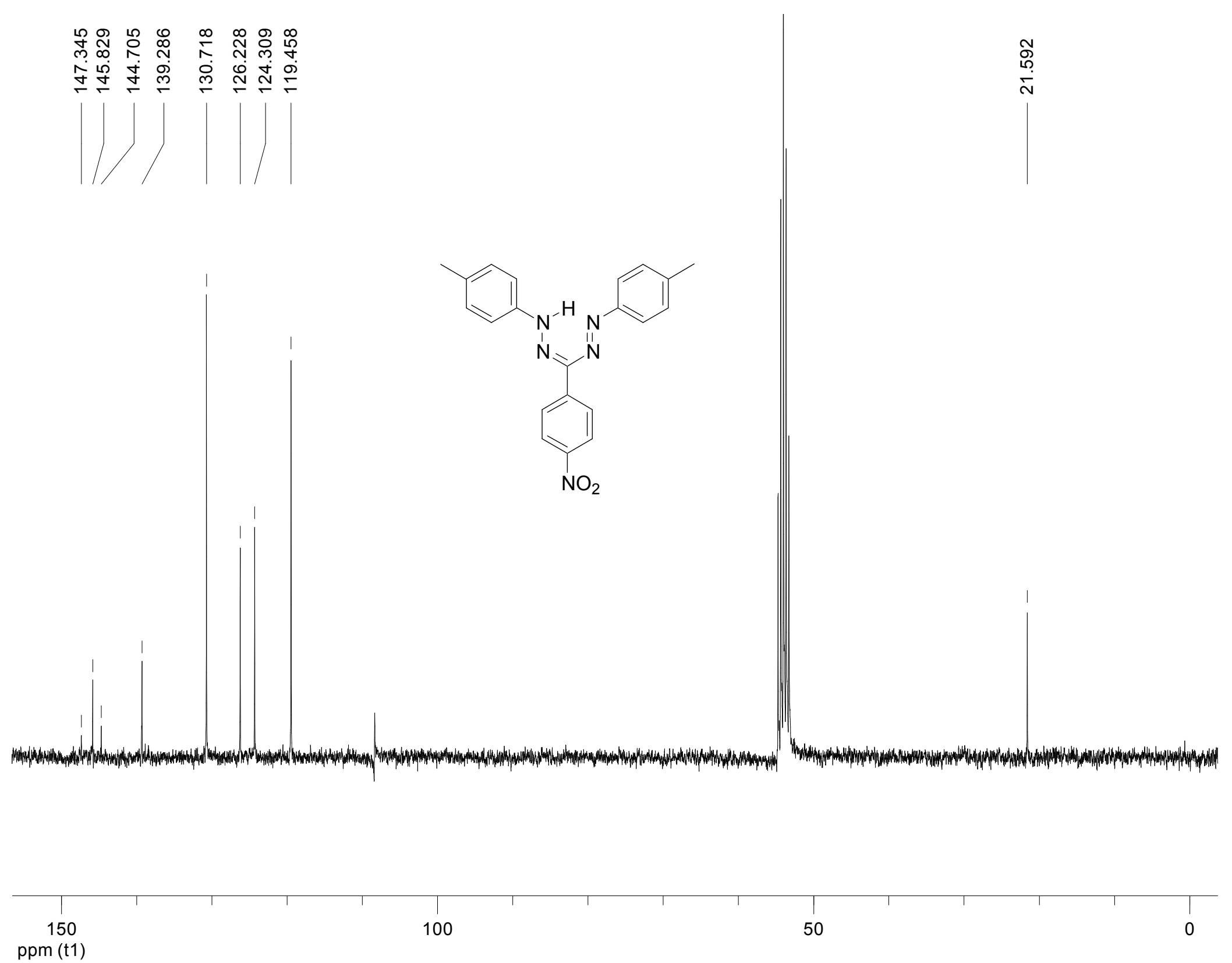

Figure S18. ${ }^{13} \mathrm{C}$ NMR of $4 \mathbf{k}\left(\mathrm{CD}_{2} \mathrm{Cl}_{2}\right)$ 


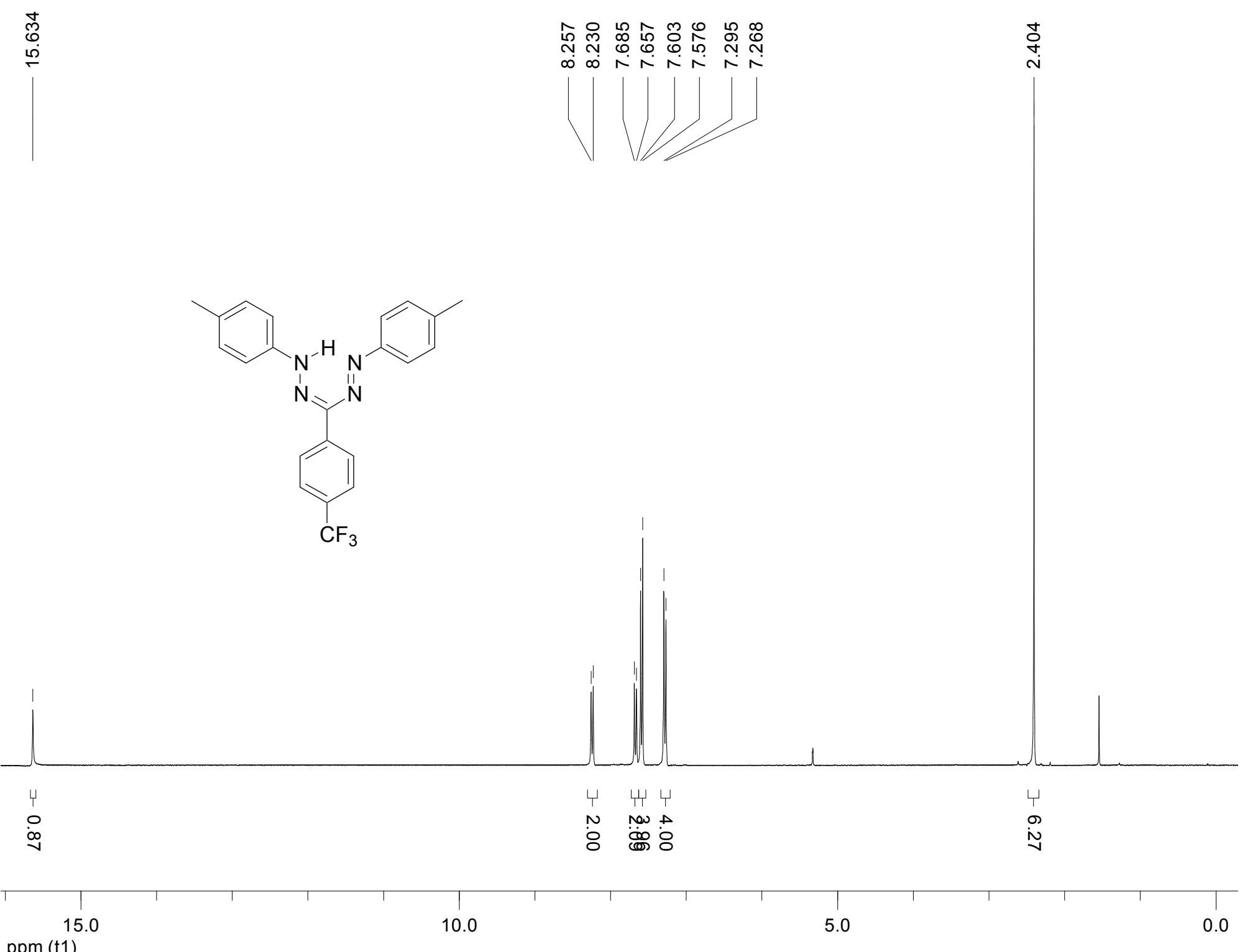

Figure S19. ${ }^{1} \mathrm{H}$ NMR of $4 \mathbf{l}\left(\mathrm{CD}_{2} \mathrm{Cl}_{2}\right)$ 


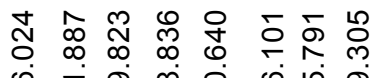

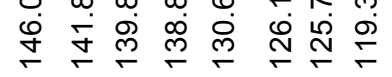
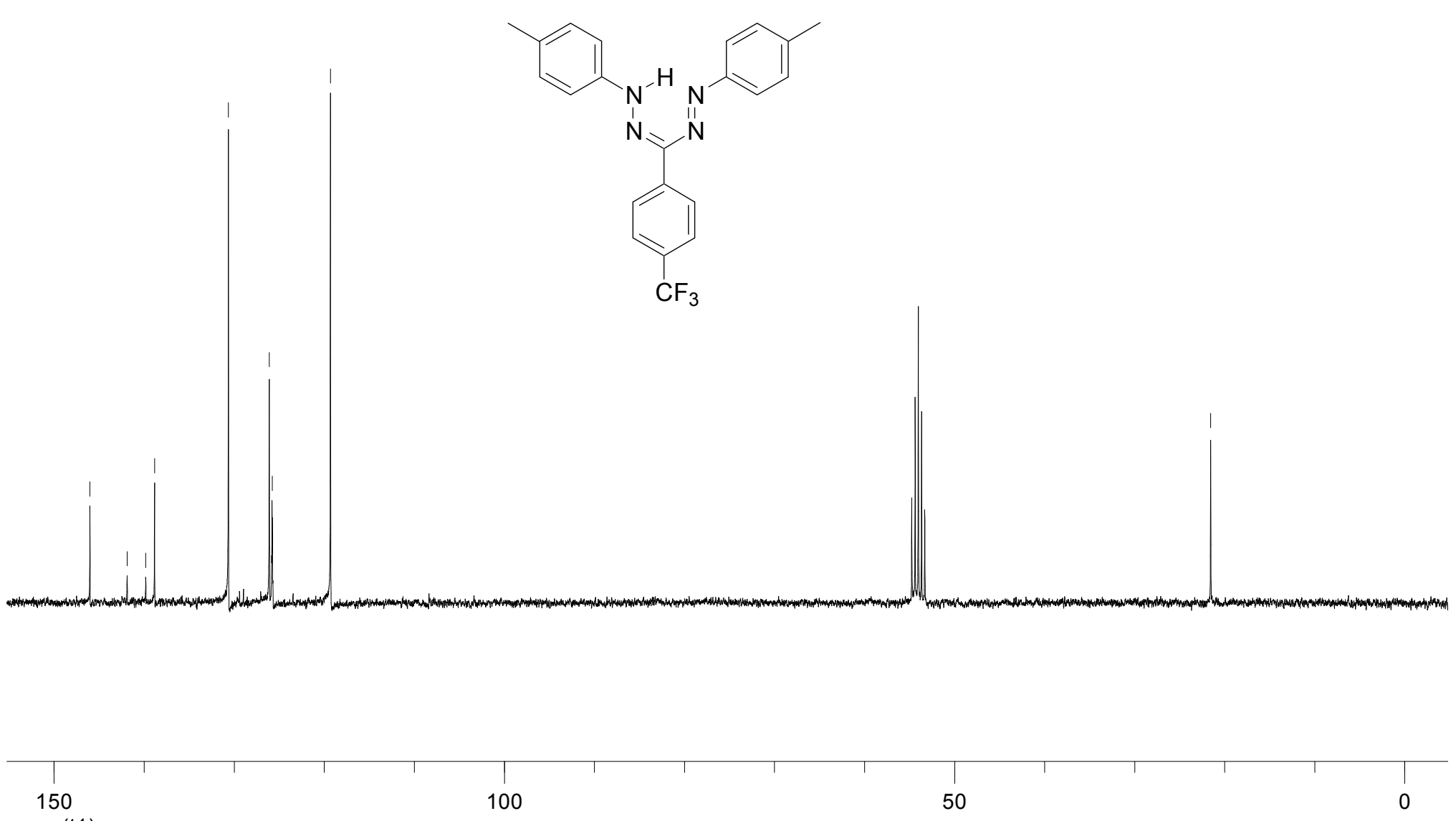

ppm (t1)

Figure S20. ${ }^{13} \mathrm{C}$ NMR of $4 \mathbf{l}\left(\mathrm{CD}_{2} \mathrm{Cl}_{2}\right)$ 


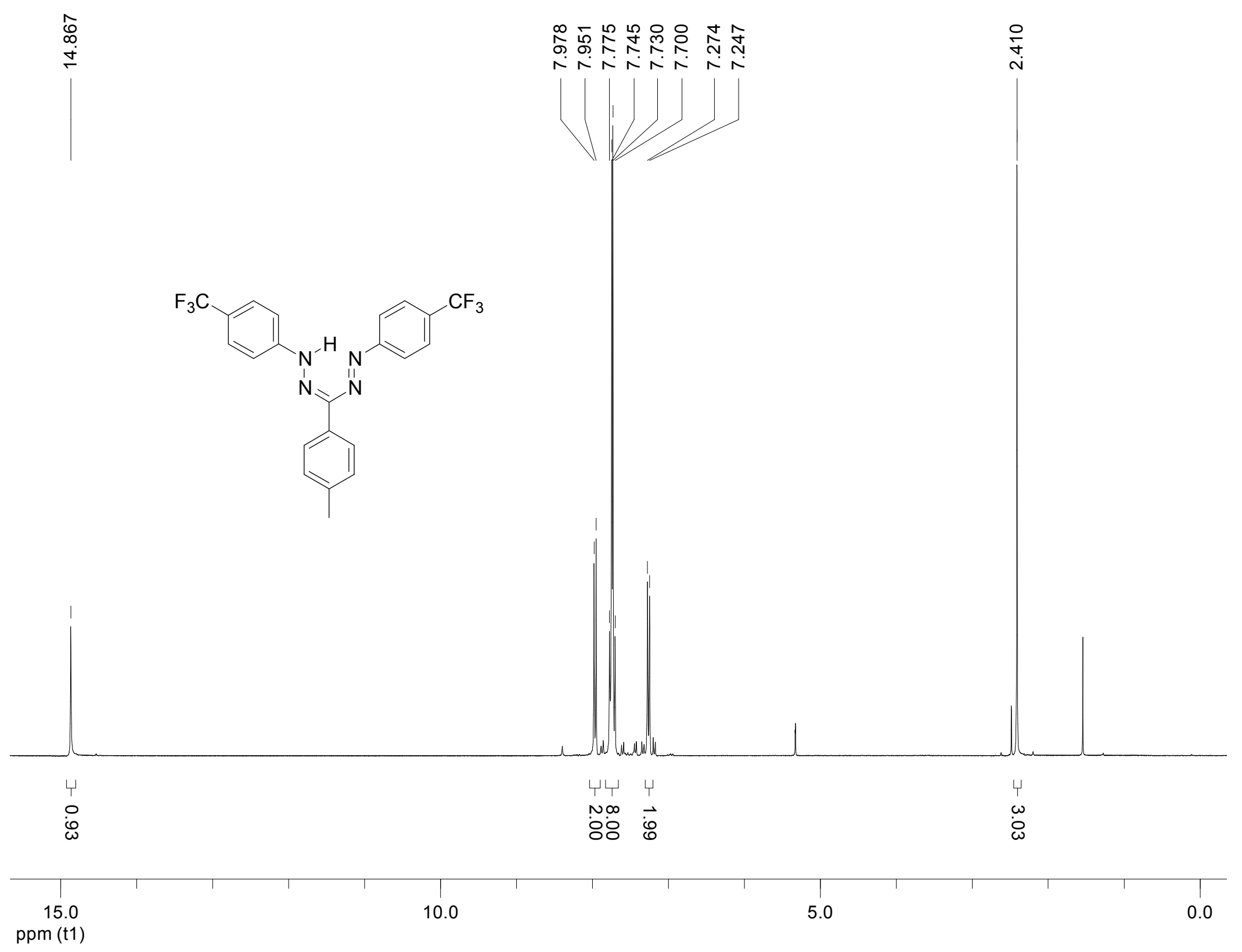

Figure S21. ${ }^{1} \mathrm{H} \mathrm{NMR}$ of $\mathbf{4 m}\left(\mathrm{CD}_{2} \mathrm{Cl}_{2}\right)$ 

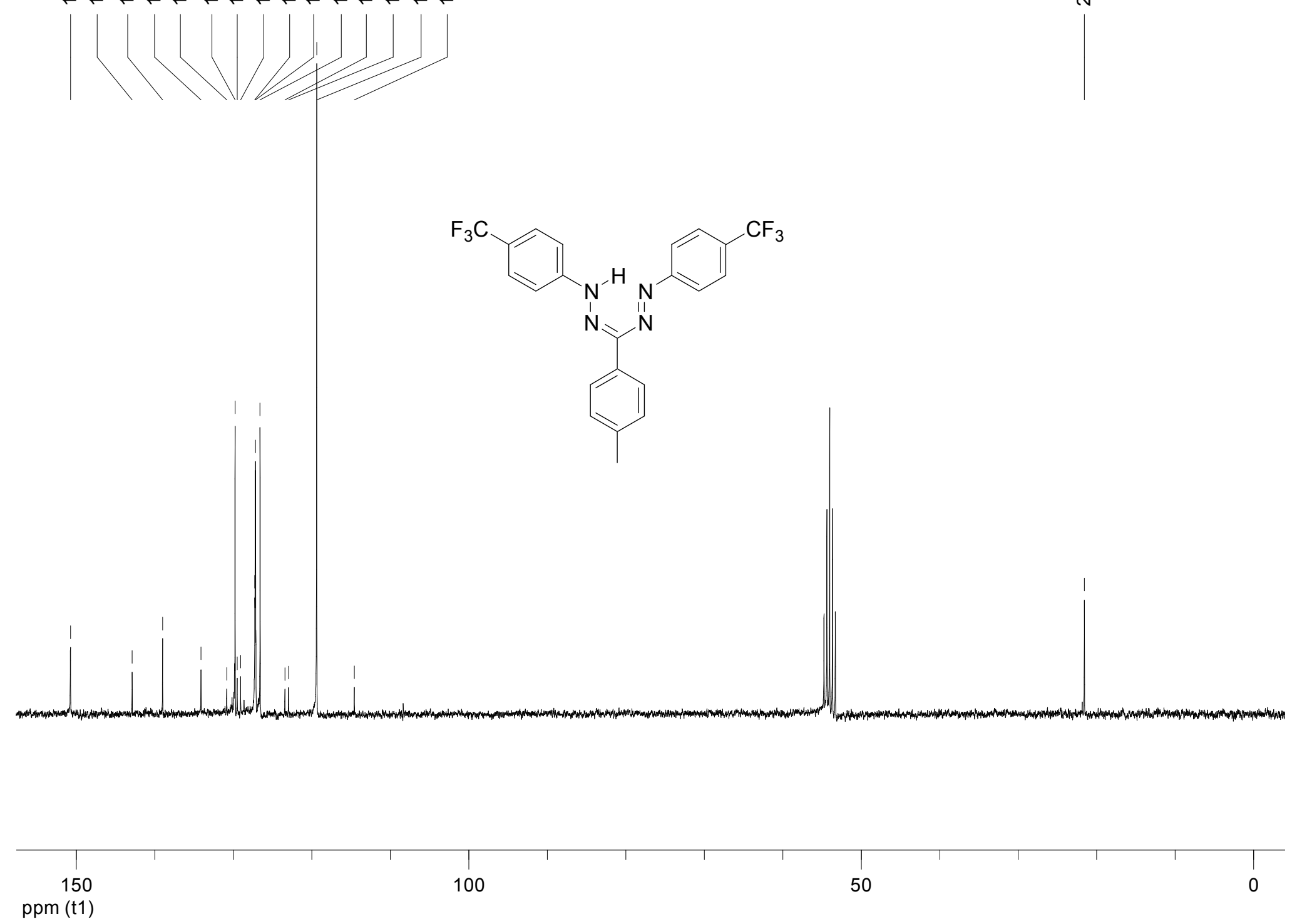

Figure S22. ${ }^{13} \mathrm{C}$ NMR of $\mathbf{4 m}\left(\mathrm{CD}_{2} \mathrm{Cl}_{2}\right)$ 


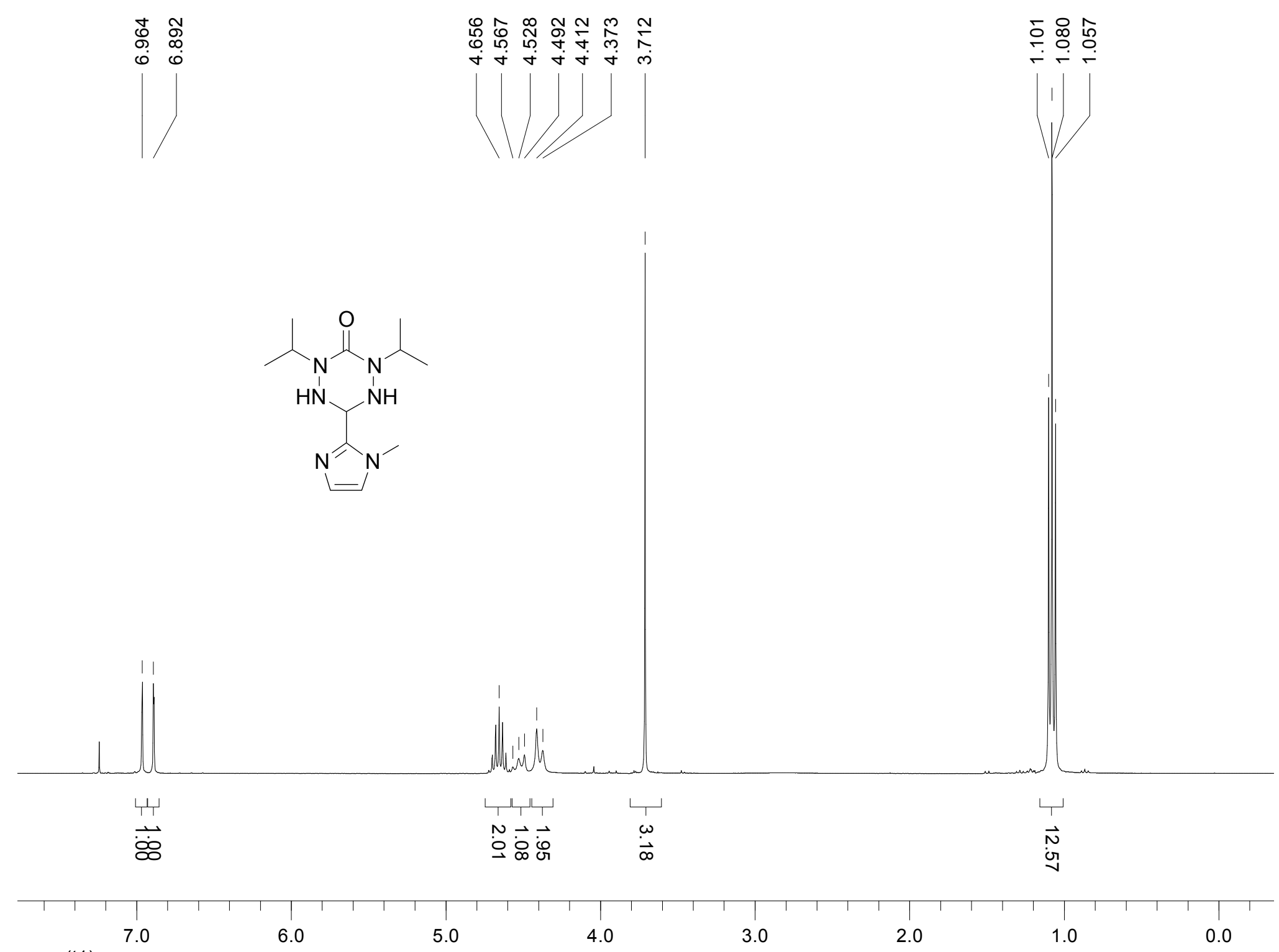

ppm (t1)

Figure S23. ${ }^{1} \mathrm{H}$ NMR of $8\left(\mathrm{CDCl}_{3}\right)$ 

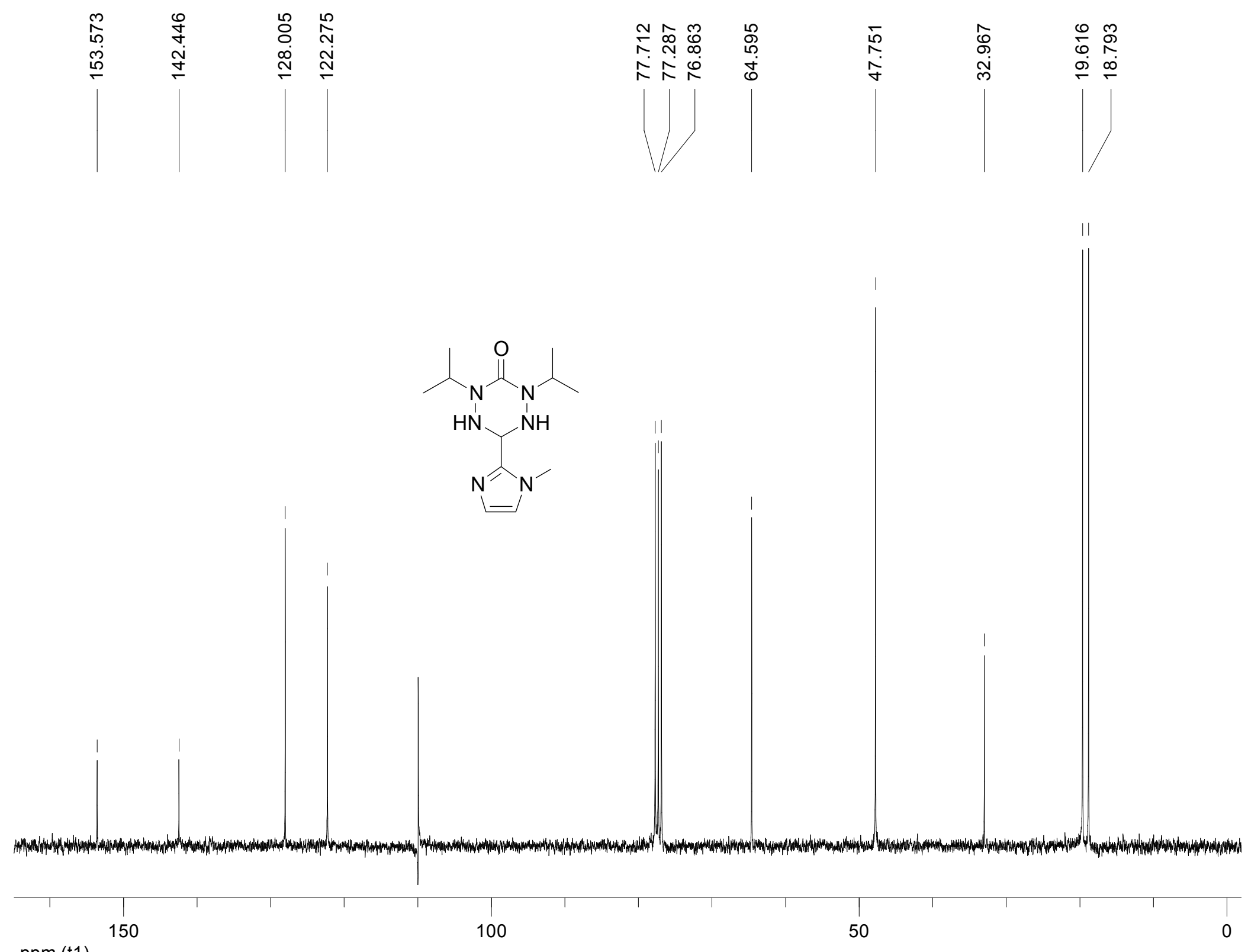

ppm (t1)

Figure S24. ${ }^{13} \mathrm{C} \mathrm{NMR}$ of $\mathbf{8}\left(\mathrm{CDCl}_{3}\right)$ 


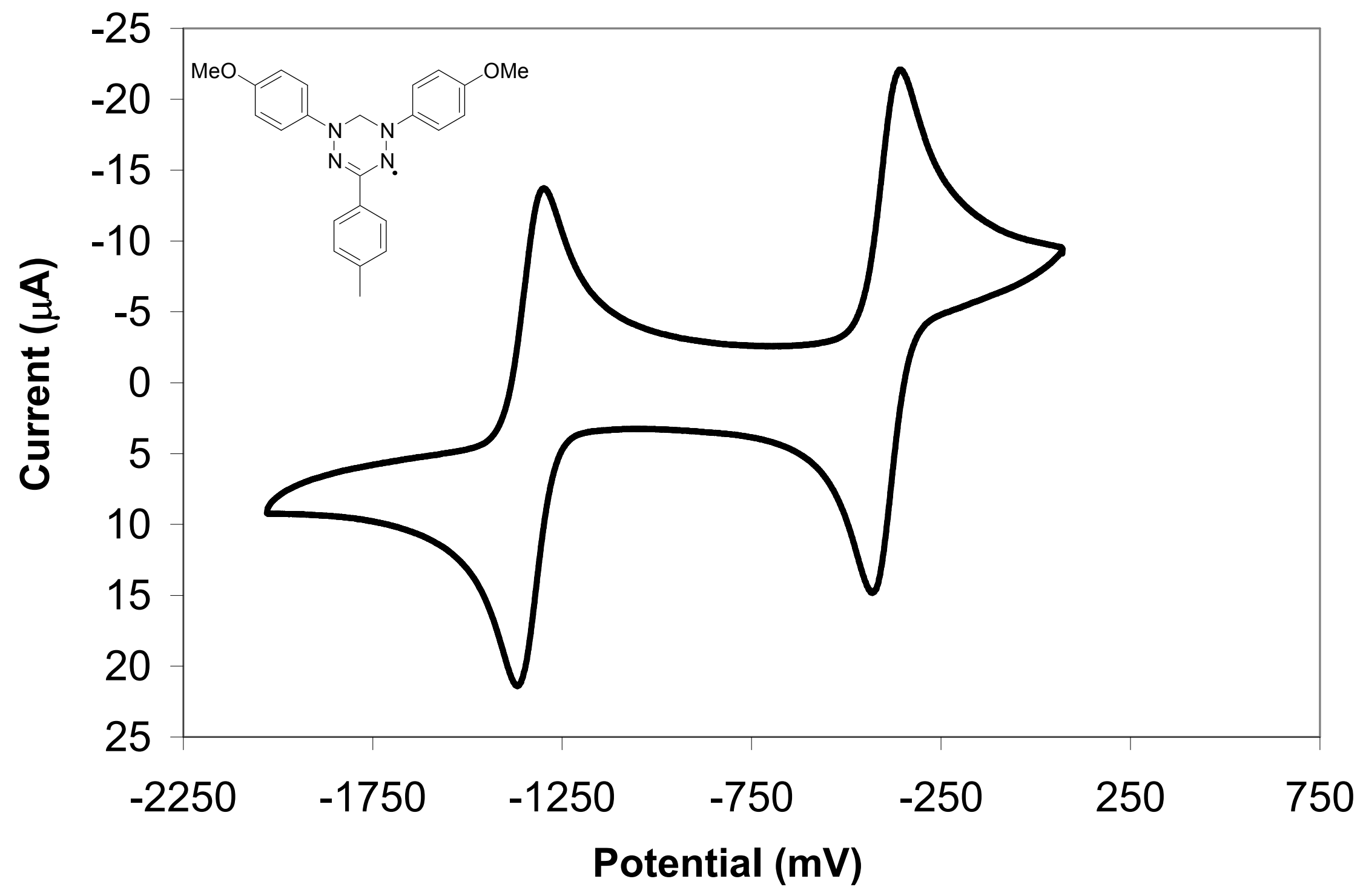

Figure S25. Cyclic Voltammogram of $\mathbf{1 b}$ 


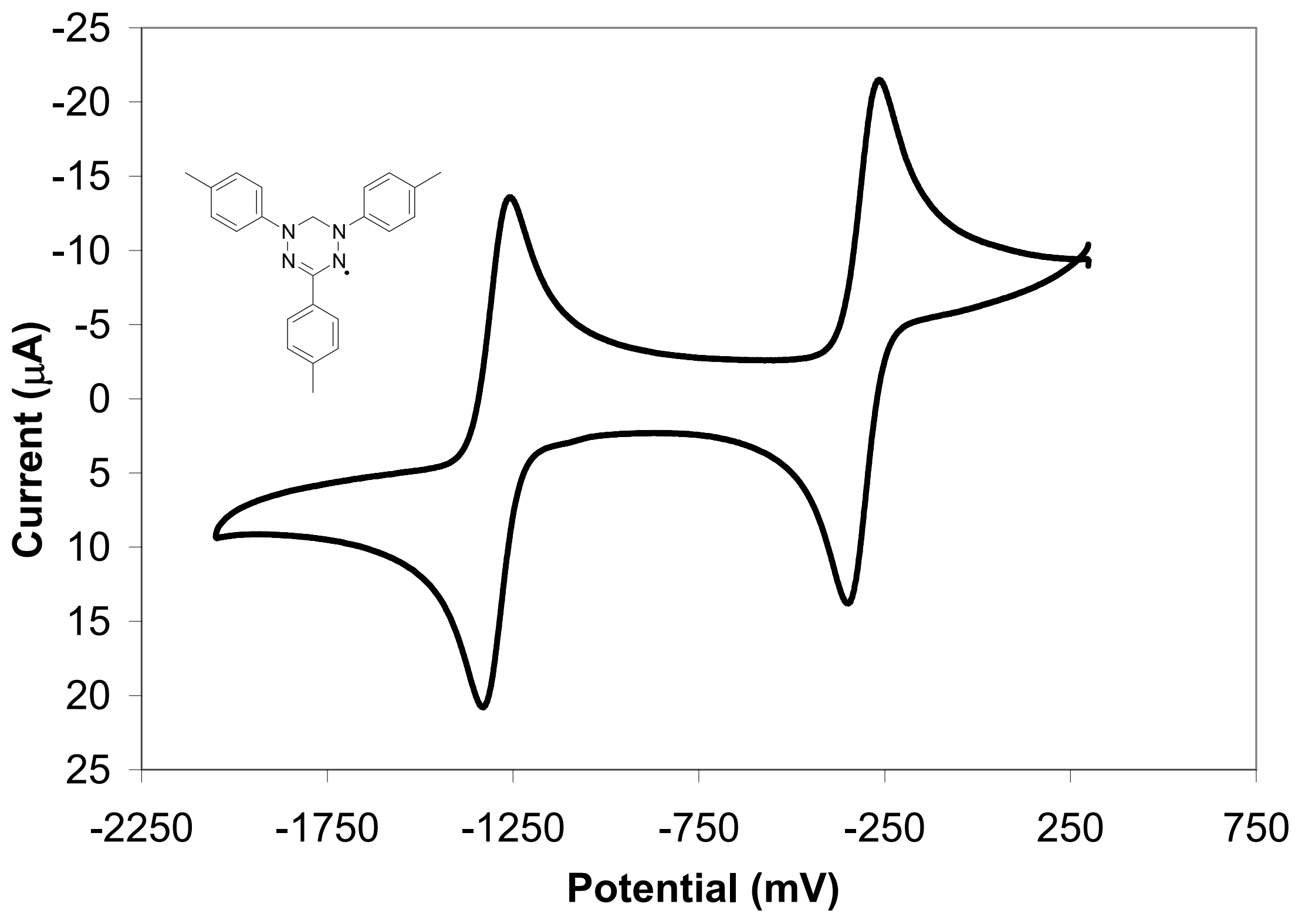

Figure S26. Cyclic Voltammogram of 1c 


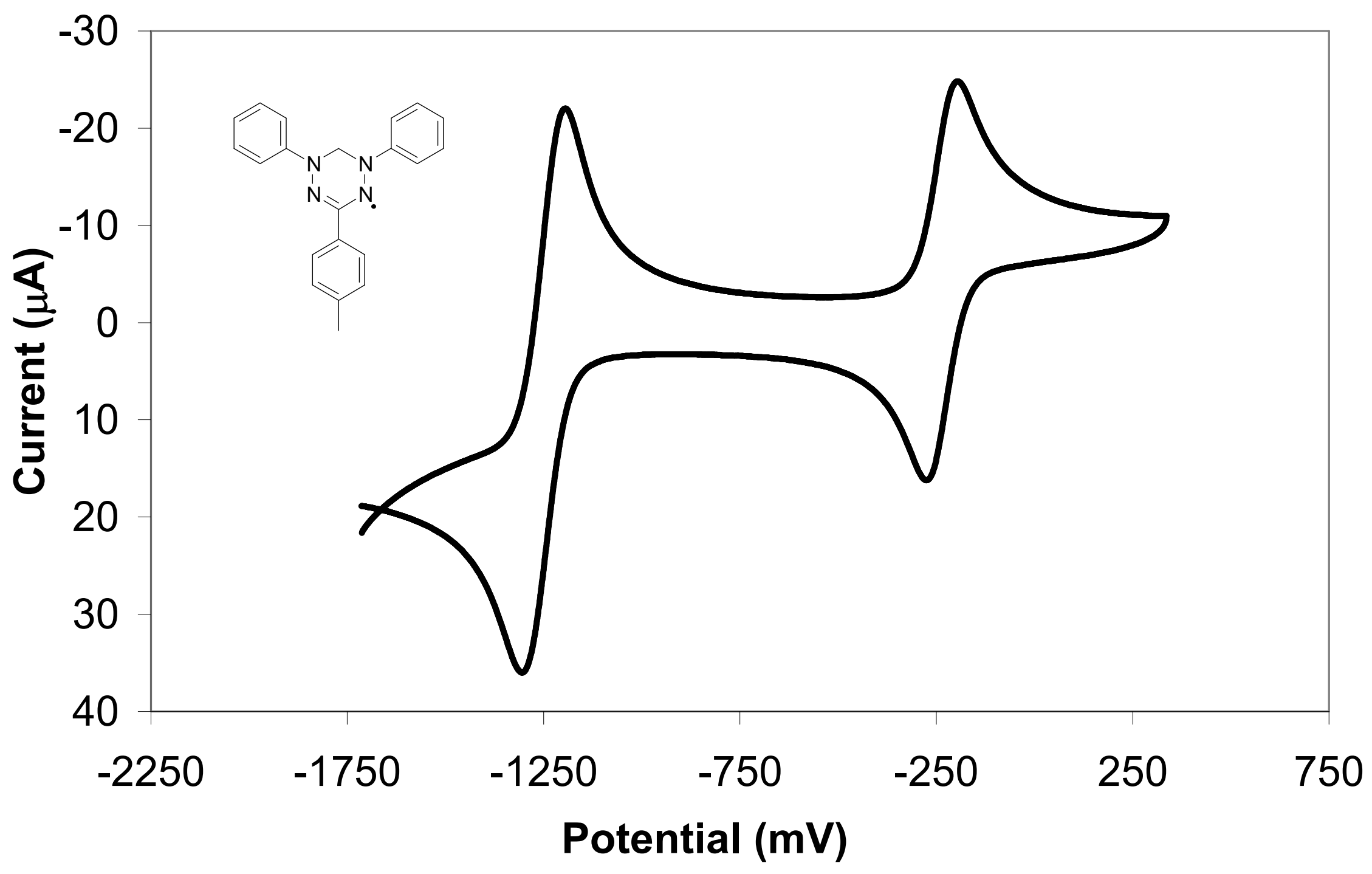

Figure S27. Cyclic Voltammogram of 1d 


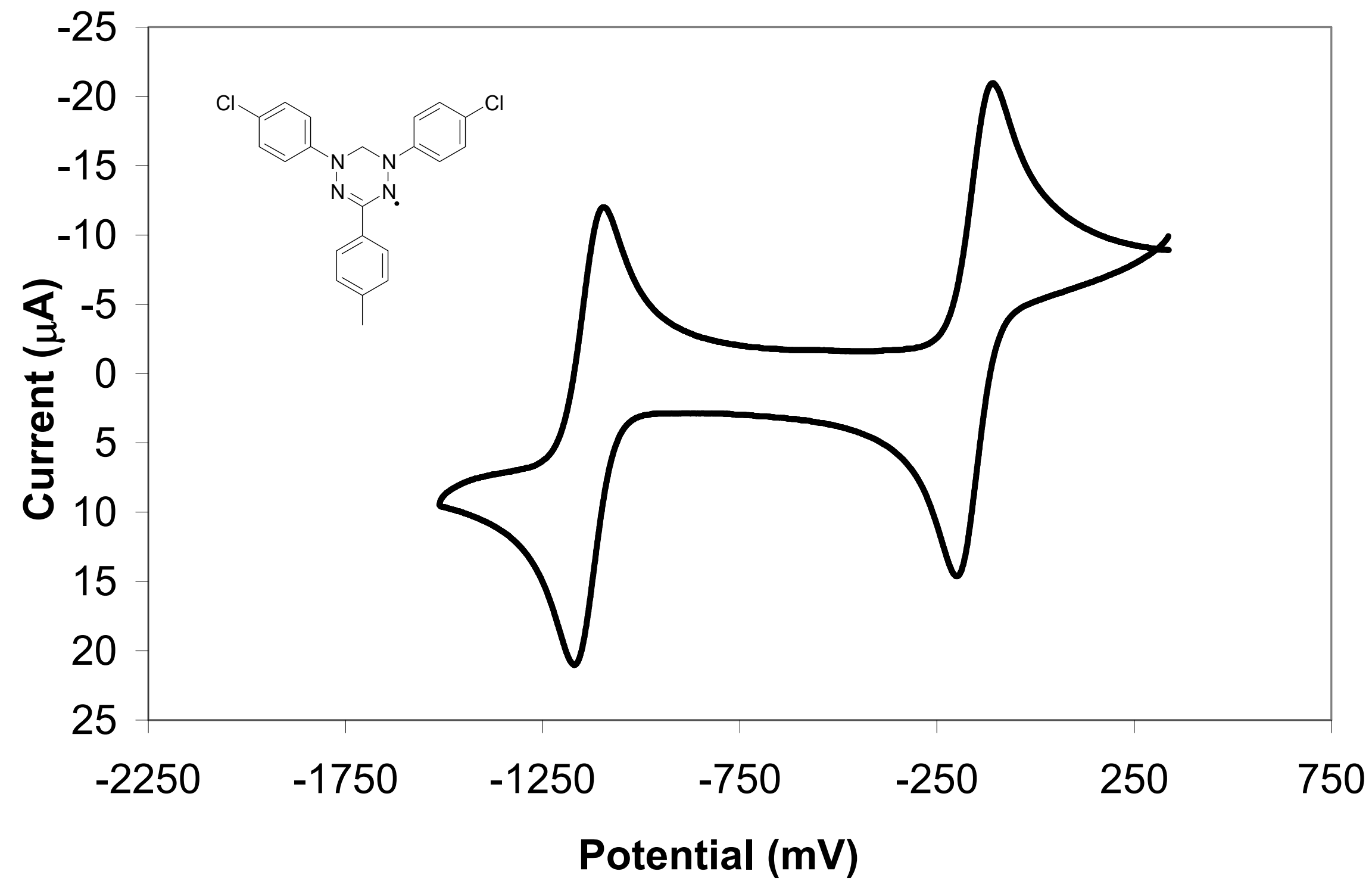

Figure S28. Cyclic Voltammogram of 1e 


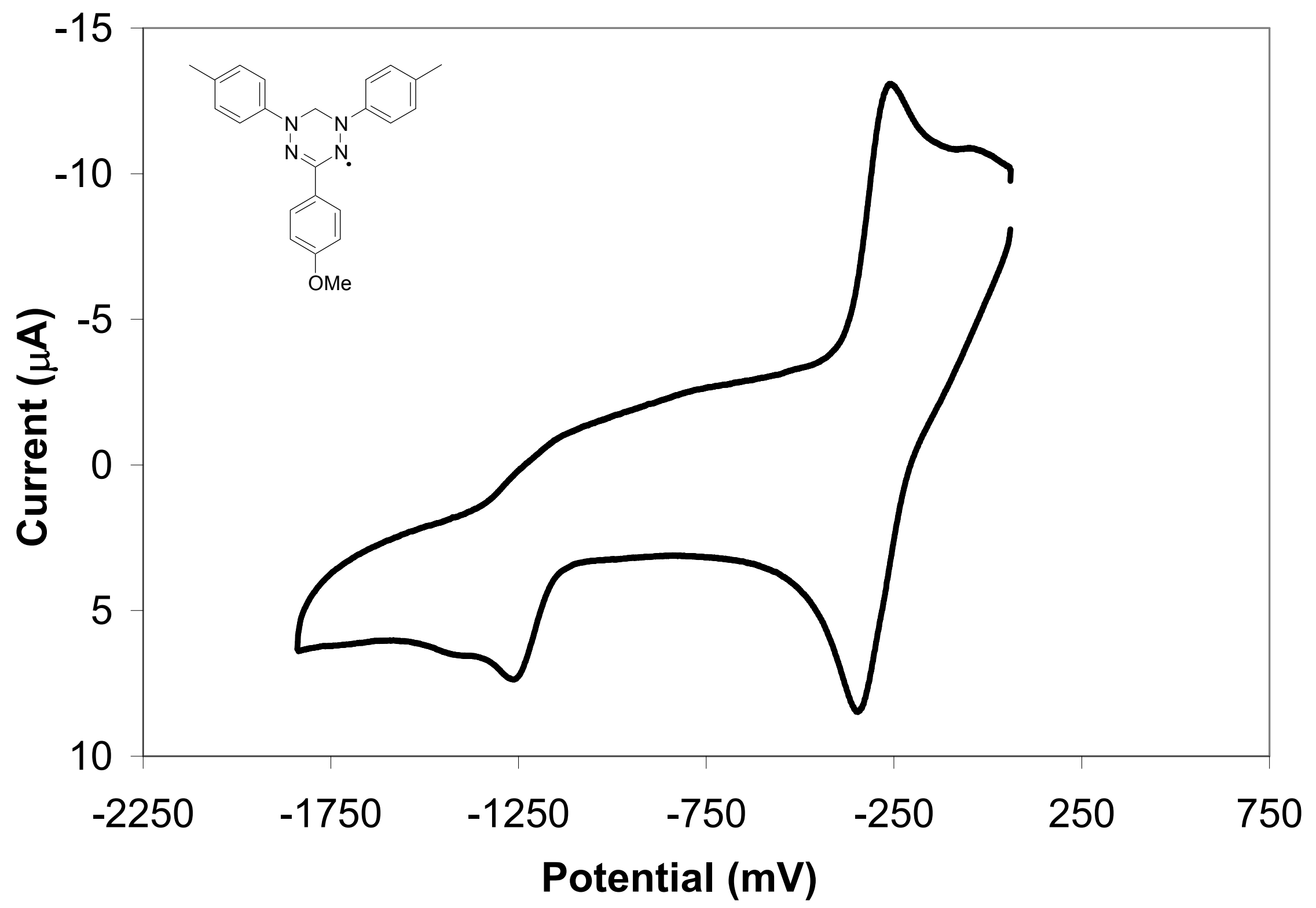

Figure S29. Cyclic Voltammogram of $\mathbf{1 g}$ 


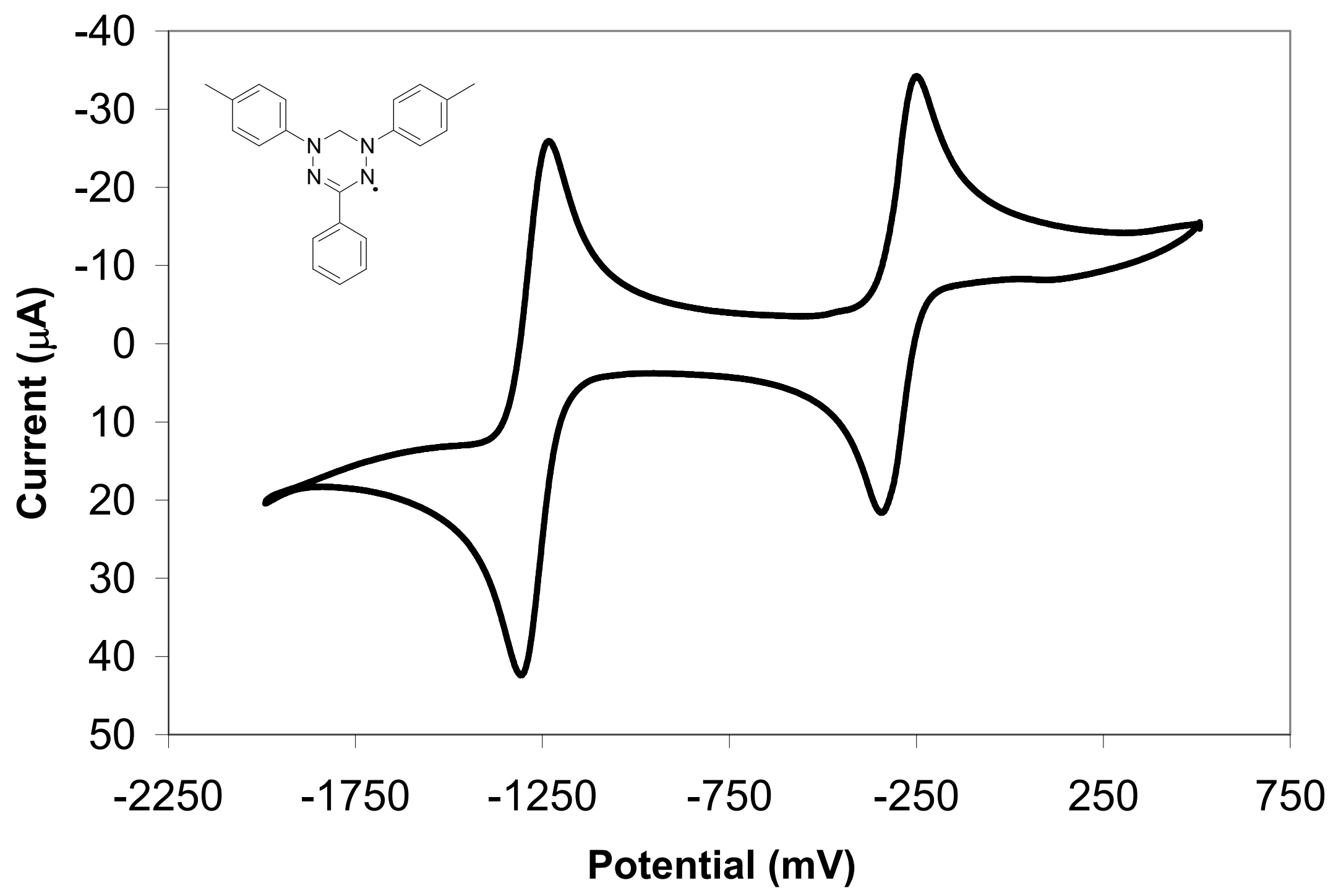

Figure S30. Cyclic Voltammogram of $\mathbf{1 h}$ 


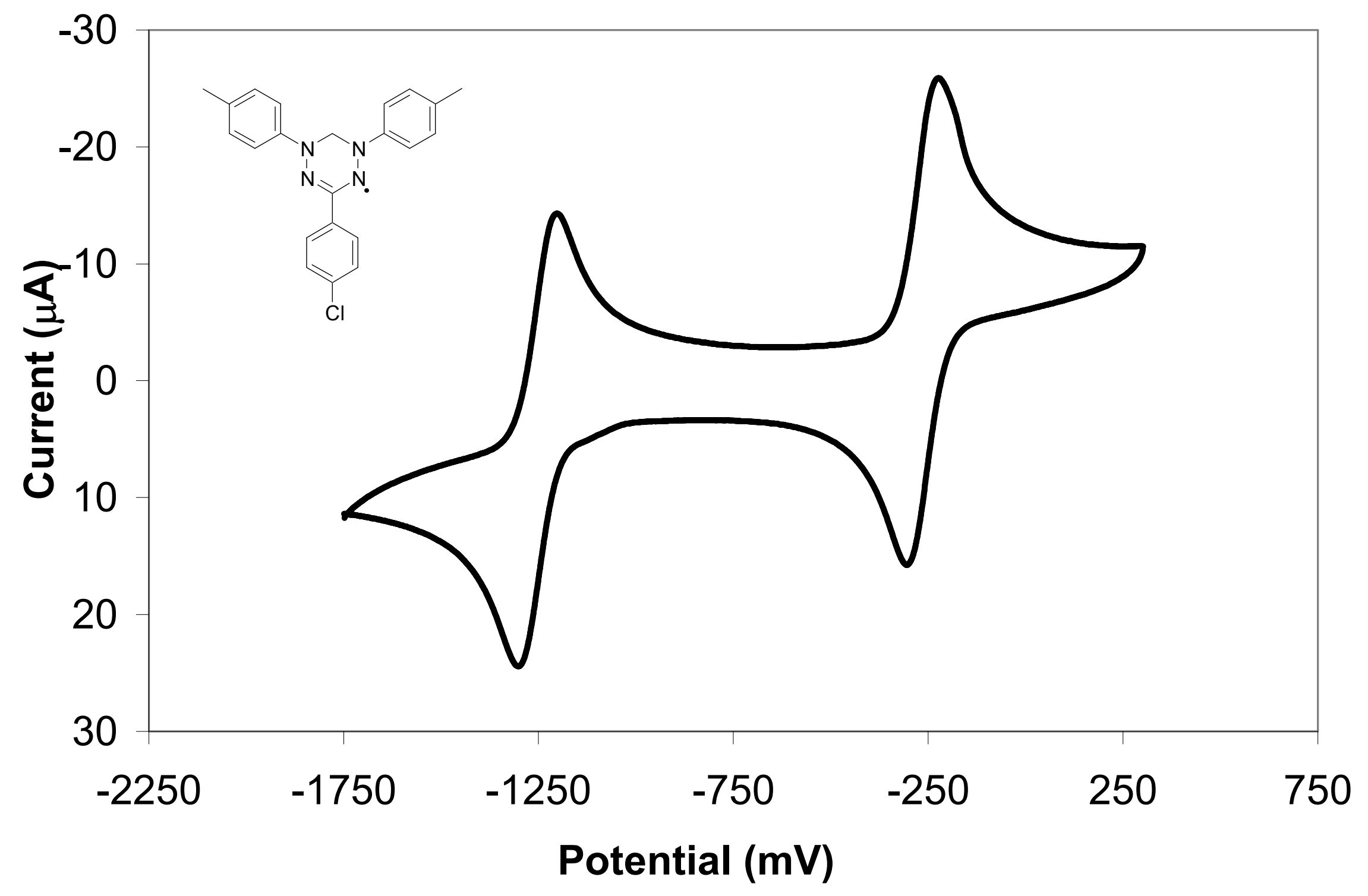

Figure S31. Cyclic Voltammogram of 1i 


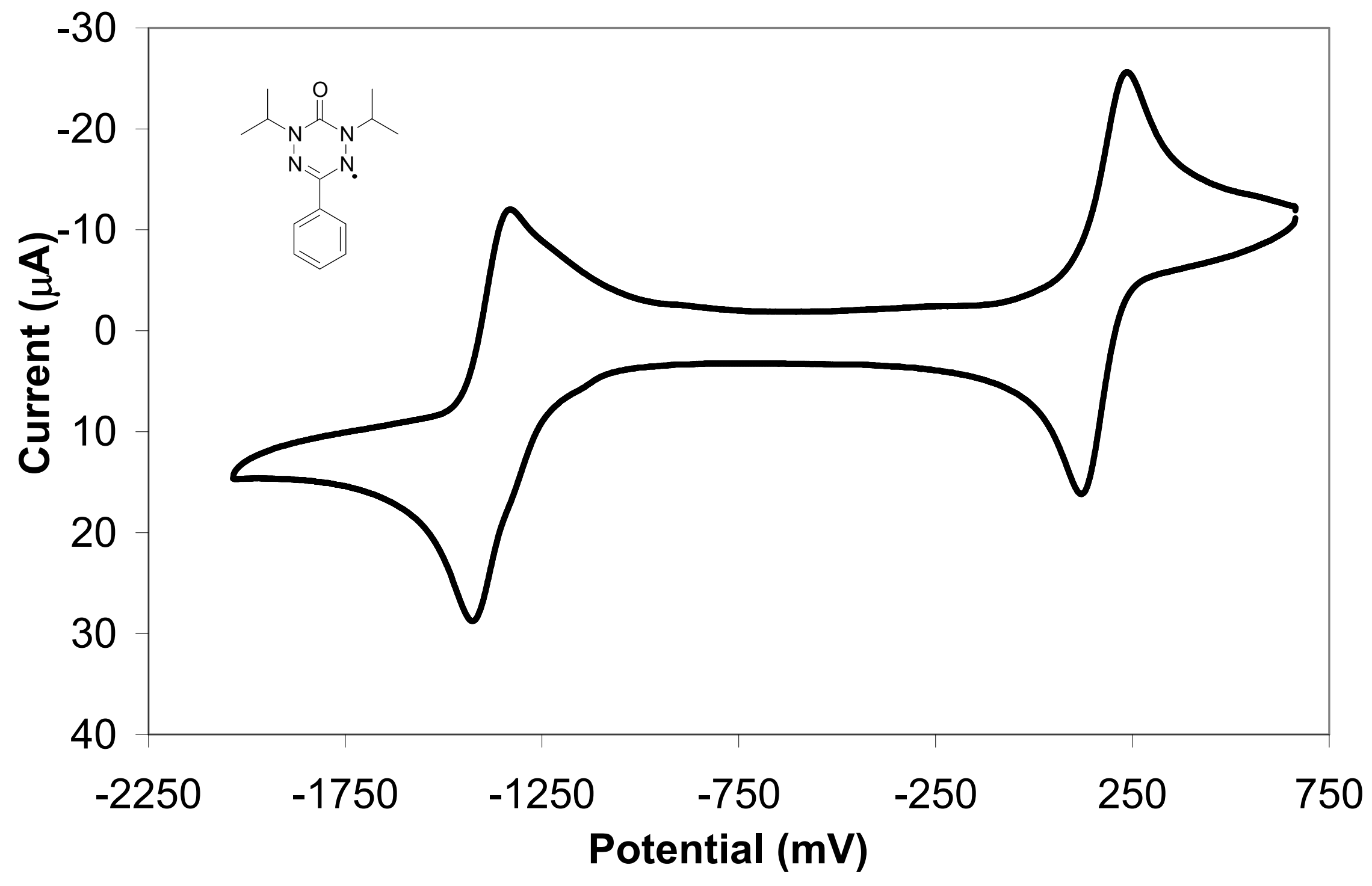

Figure S32. Cyclic Voltammogram of 2c 


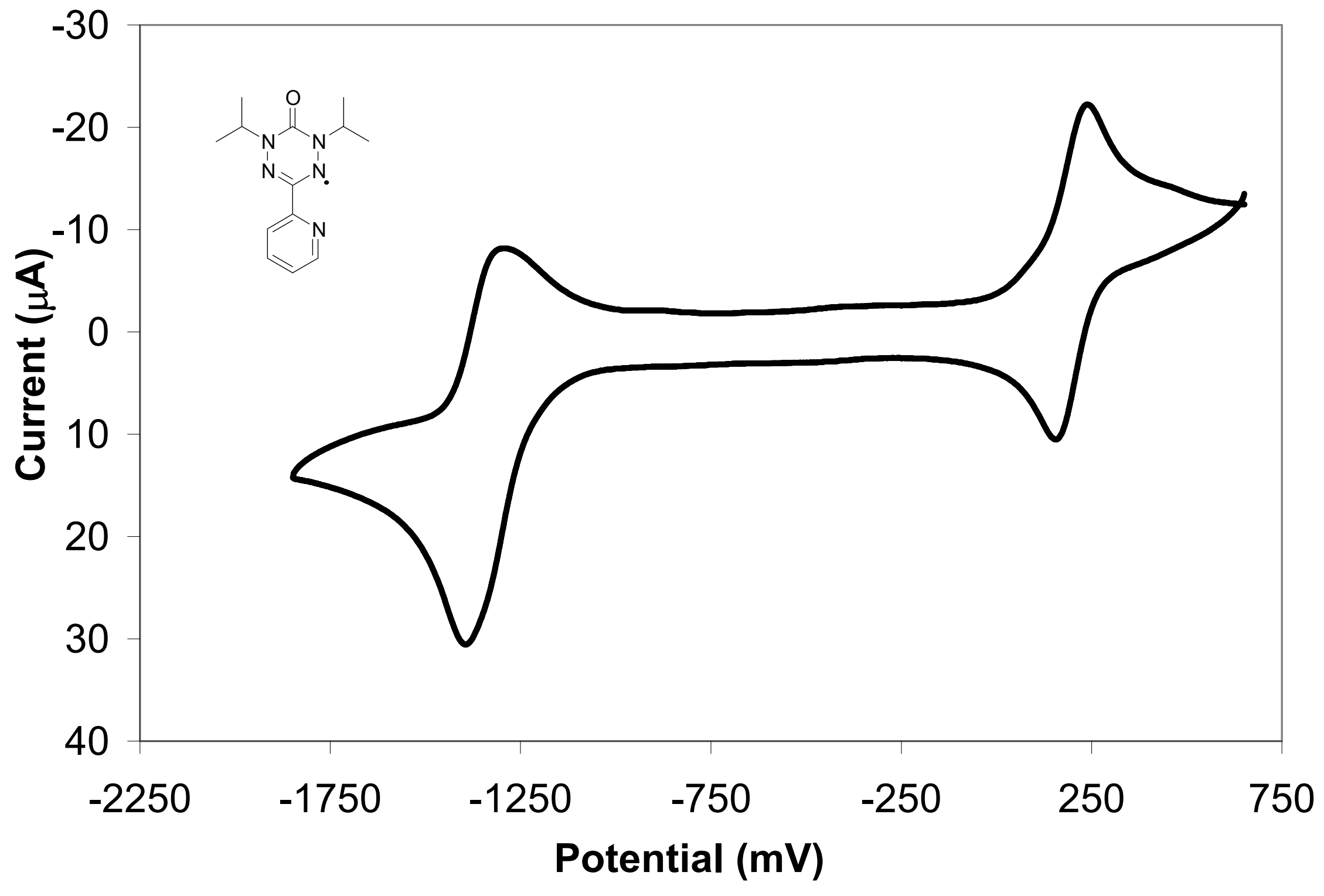

Figure S33. Cyclic Voltammogram of 2d 


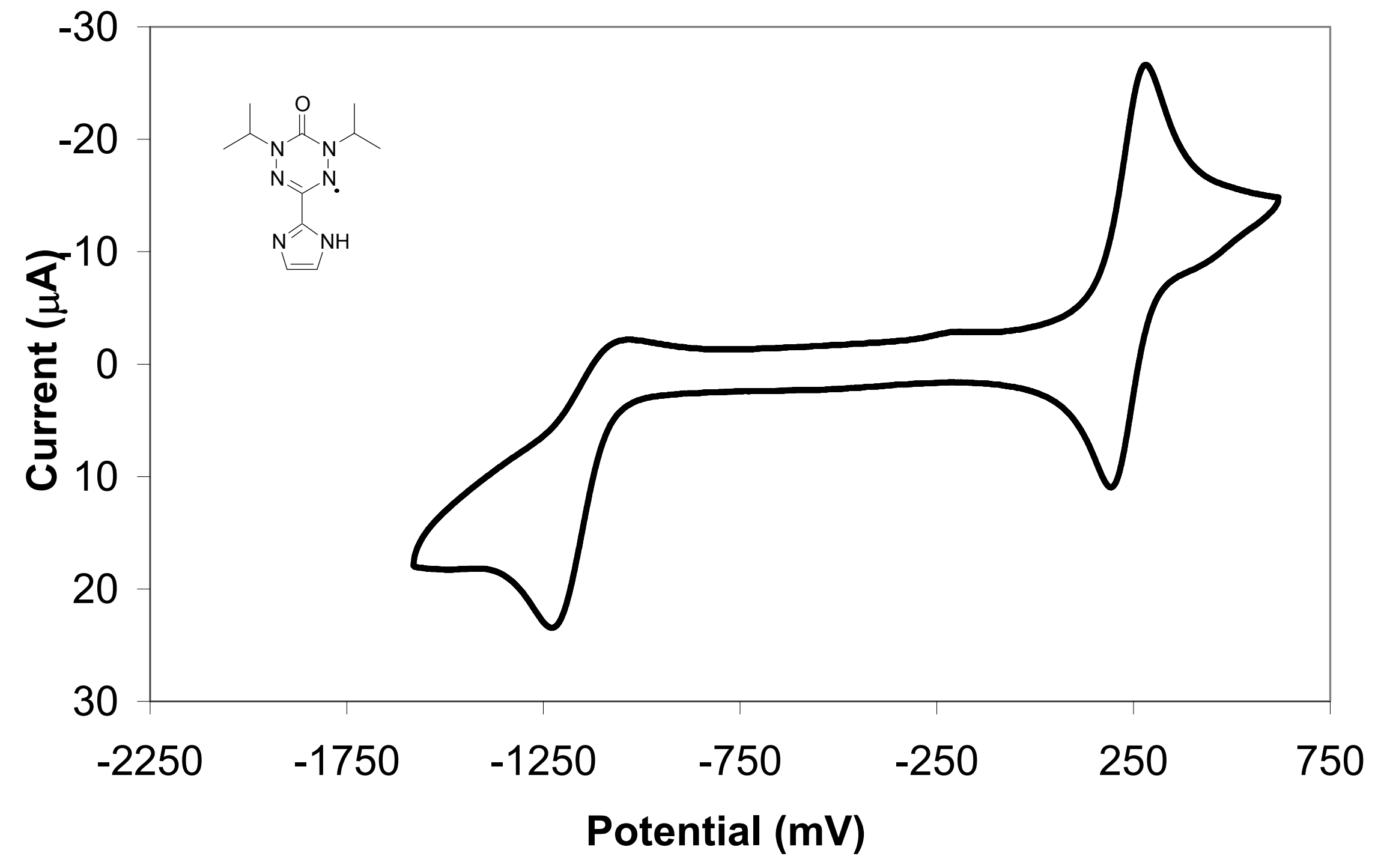

Figure S34. Cyclic Voltammogram of 2e 


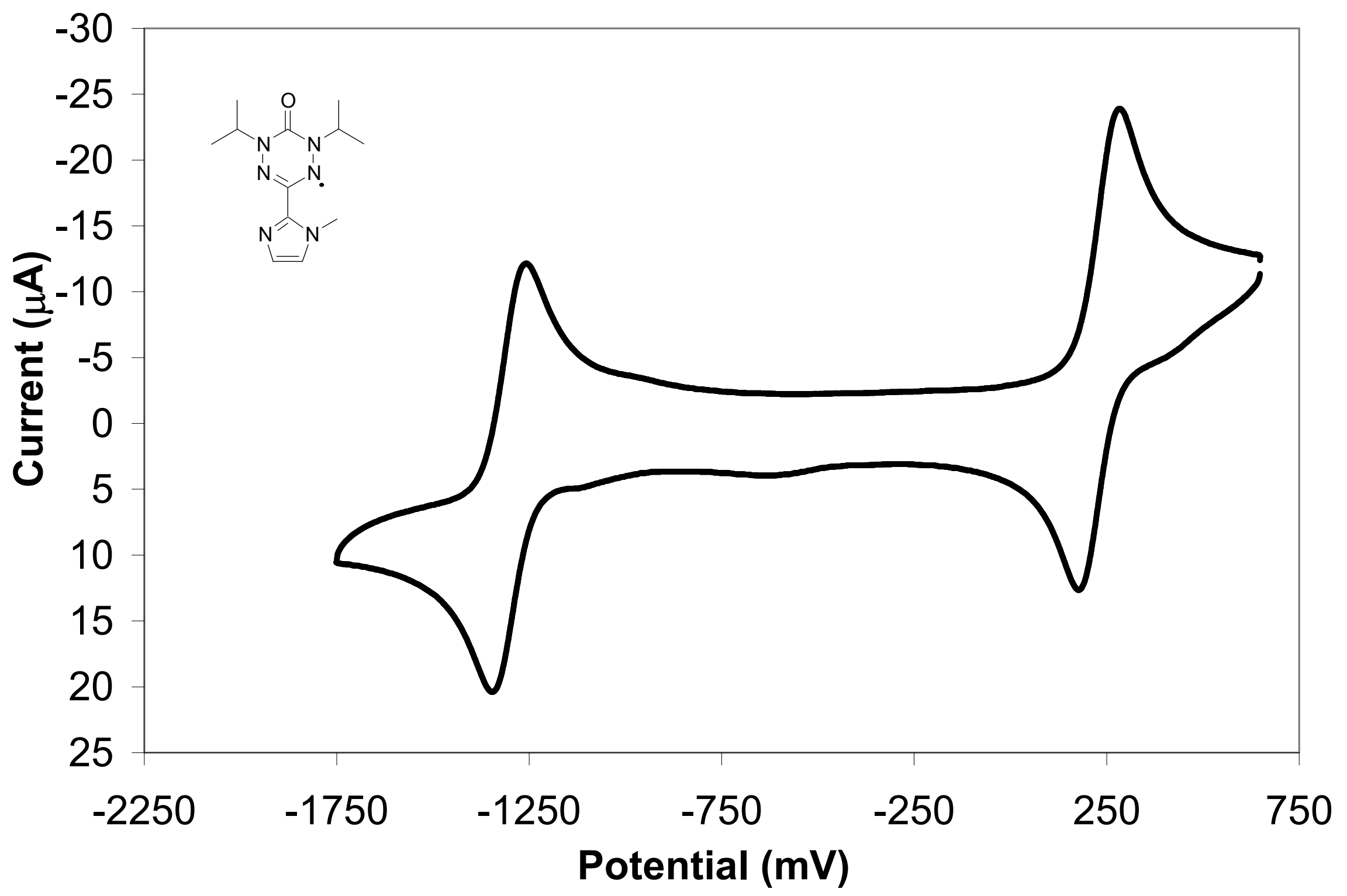

Figure S35. Cyclic Voltammogram of $\mathbf{2 f}$ 


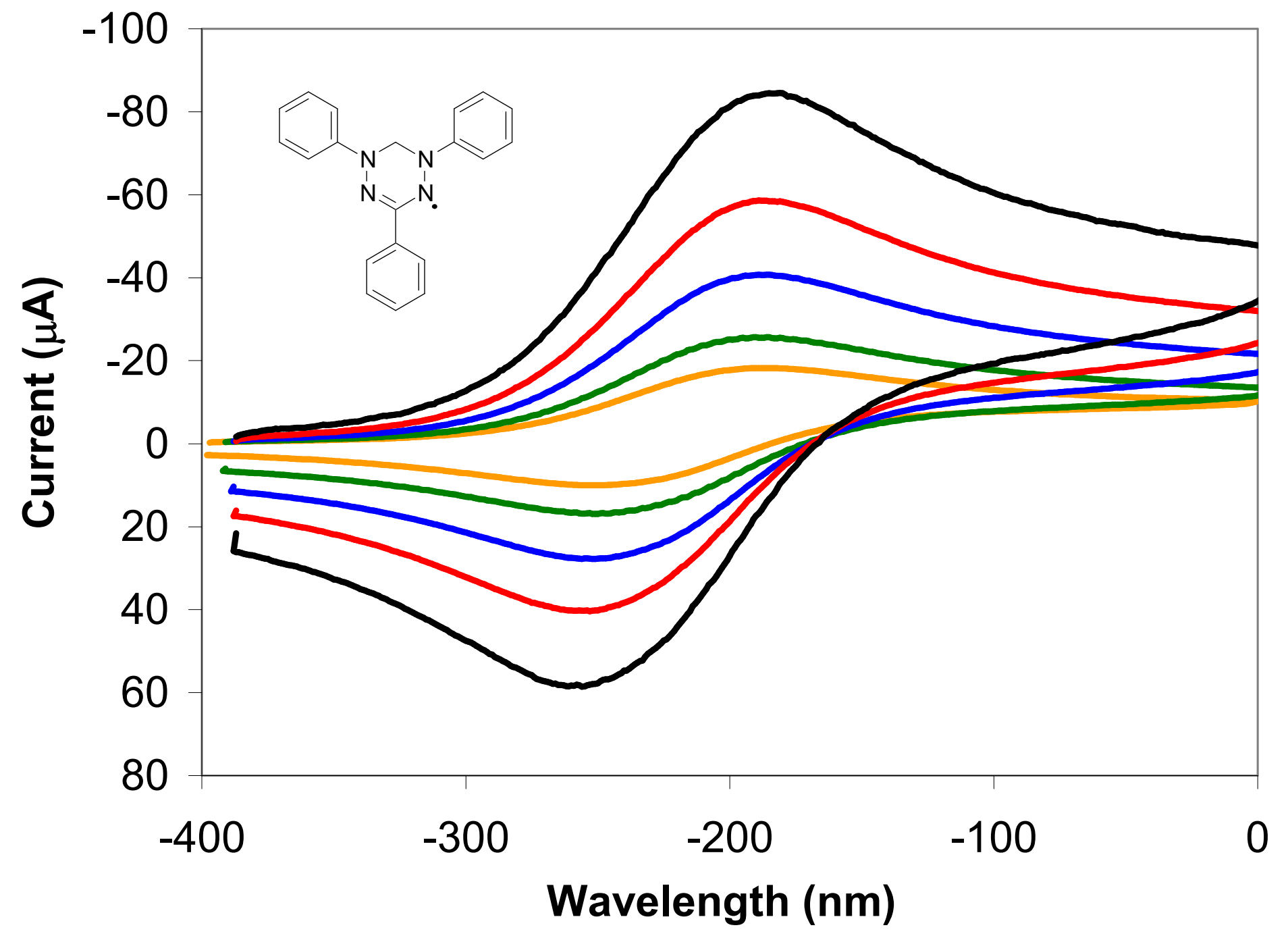

Figure S36. Oxidation wave of $\mathbf{1 a}$ at $50 \mathrm{mV} / \mathrm{s}$ (orange line), $100 \mathrm{mV} / \mathrm{s}$ (green line), $250 \mathrm{mV} / \mathrm{s}$ (blue line), $500 \mathrm{mV} / \mathrm{s}$ (red line), and $1000 \mathrm{mV} / \mathrm{s}$ (black line) 


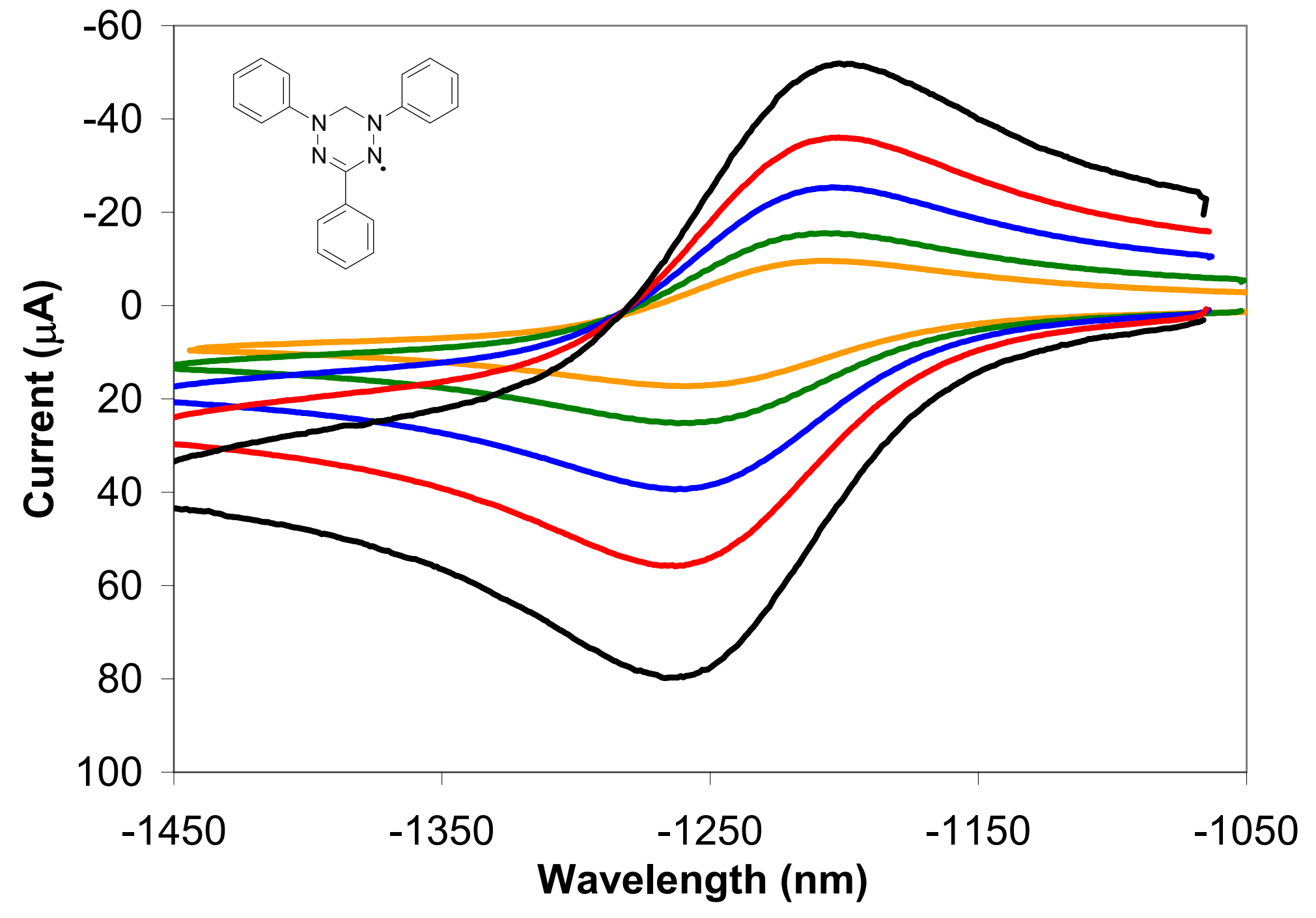

Figure S37. Reduction wave of $1 \mathbf{a}$ at $50 \mathrm{mV} / \mathrm{s}$ (orange line), $100 \mathrm{mV} / \mathrm{s}$ (green line), $250 \mathrm{mV} / \mathrm{s}$ (blue line),

$500 \mathrm{mV} / \mathrm{s}$ (red line), and $1000 \mathrm{mV} / \mathrm{s}$ (black line) 


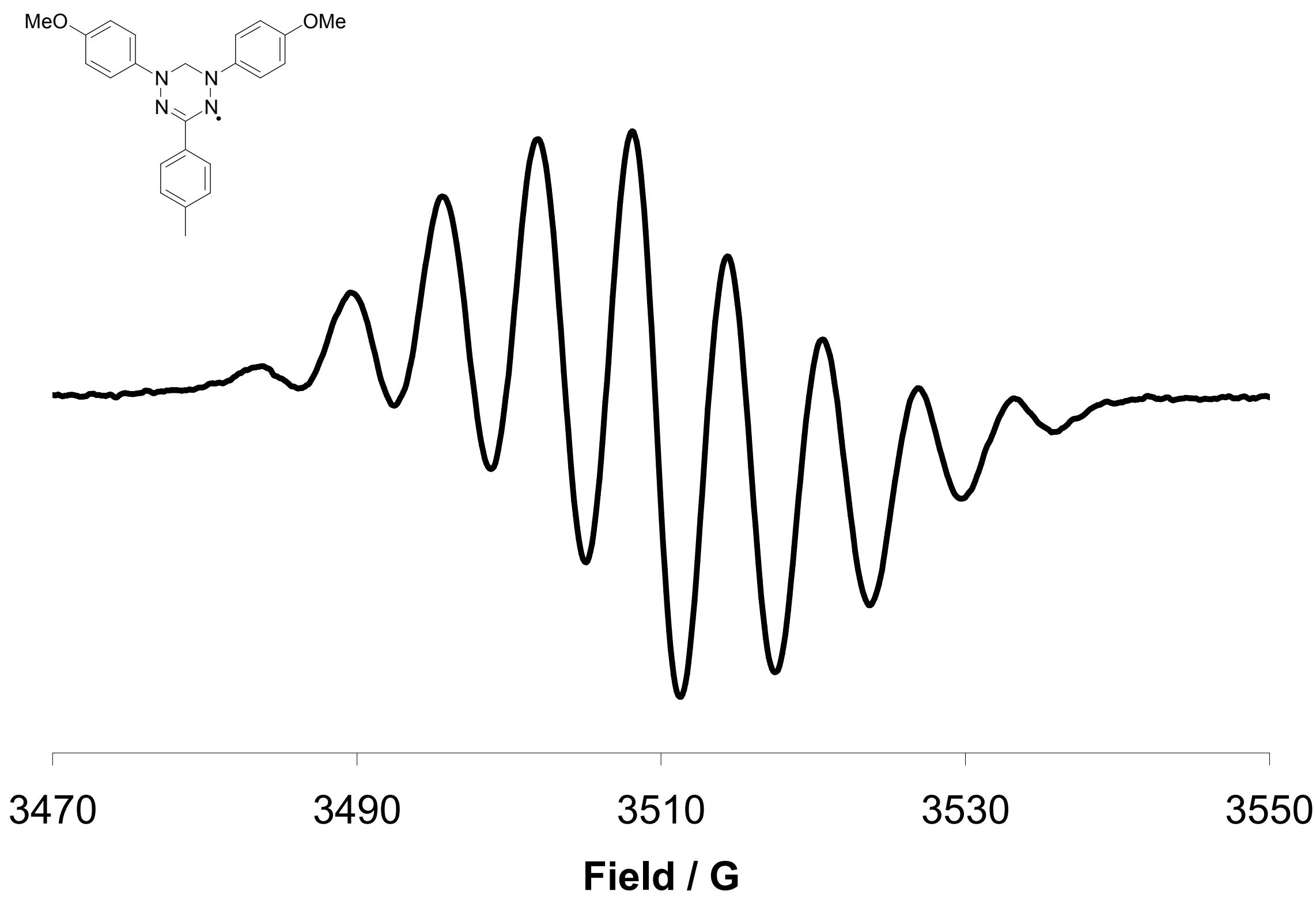

Figure S38. EPR spectrum of $\mathbf{1 b}$ in degassed $\mathrm{CH}_{2} \mathrm{Cl}_{2}(\mathrm{~g}=2.0028)$ 

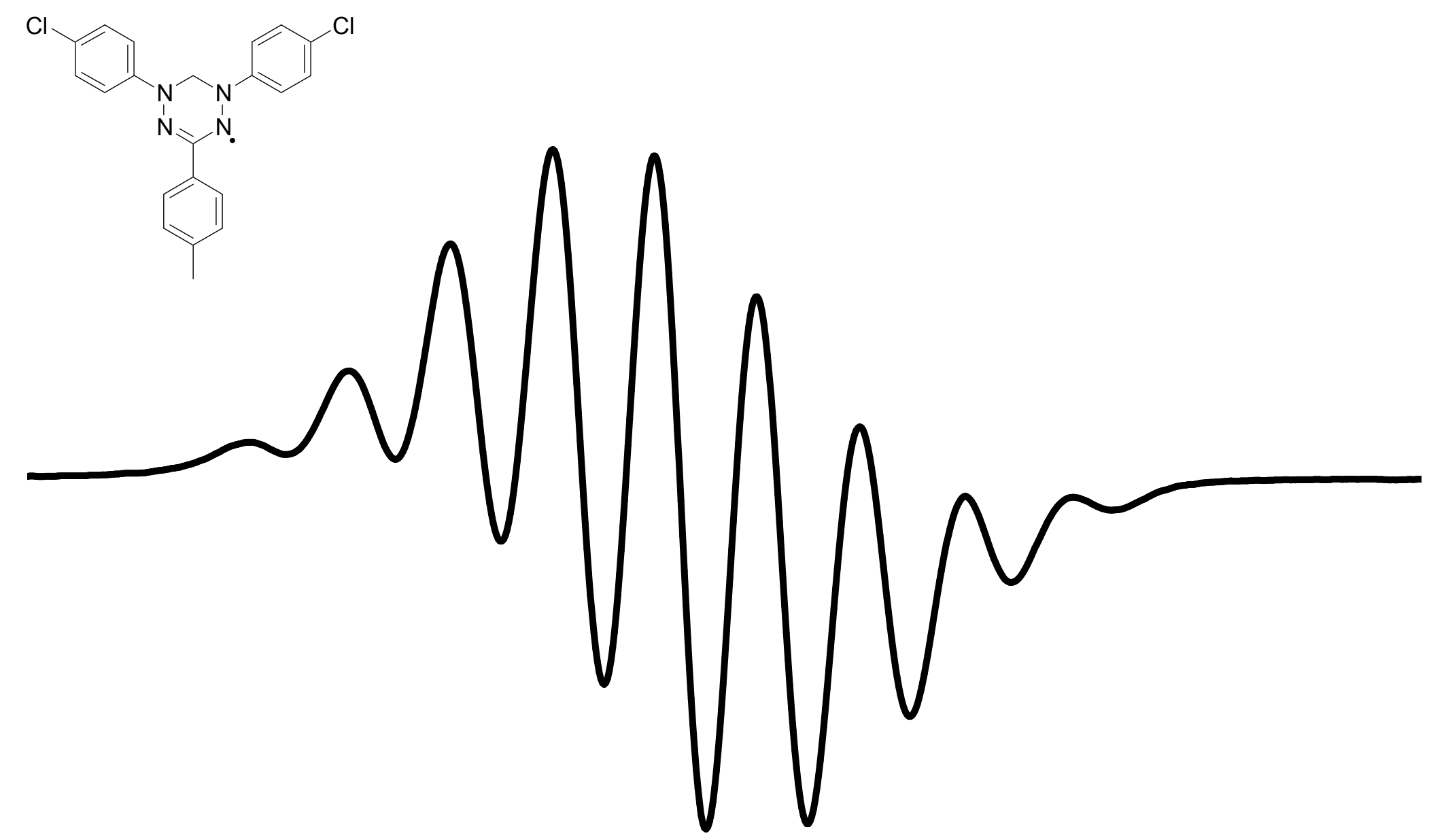

\section{Field / G}

Figure S39. EPR Spectrum of $1 \mathrm{e}$ in degassed $\mathrm{CH}_{2} \mathrm{Cl}_{2}(\mathrm{~g}=2.0035)$ 


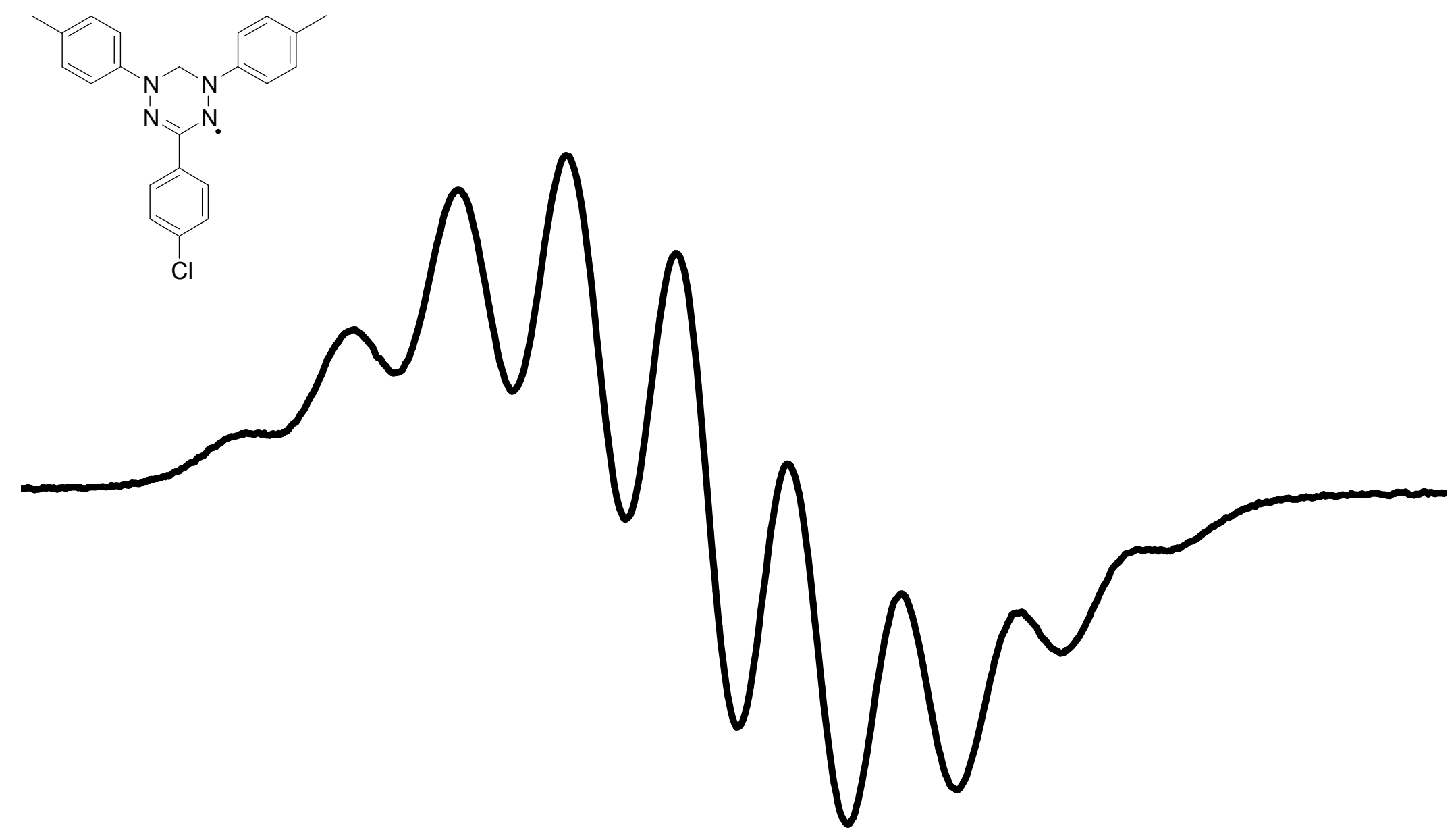

3470

3490

3510

3530

3550

Field / G

Figure S40. EPR Spectrum of $1 \mathbf{i}$ in degassed $\mathrm{CH}_{2} \mathrm{Cl}_{2}(\mathrm{~g}=2.0048)$ 


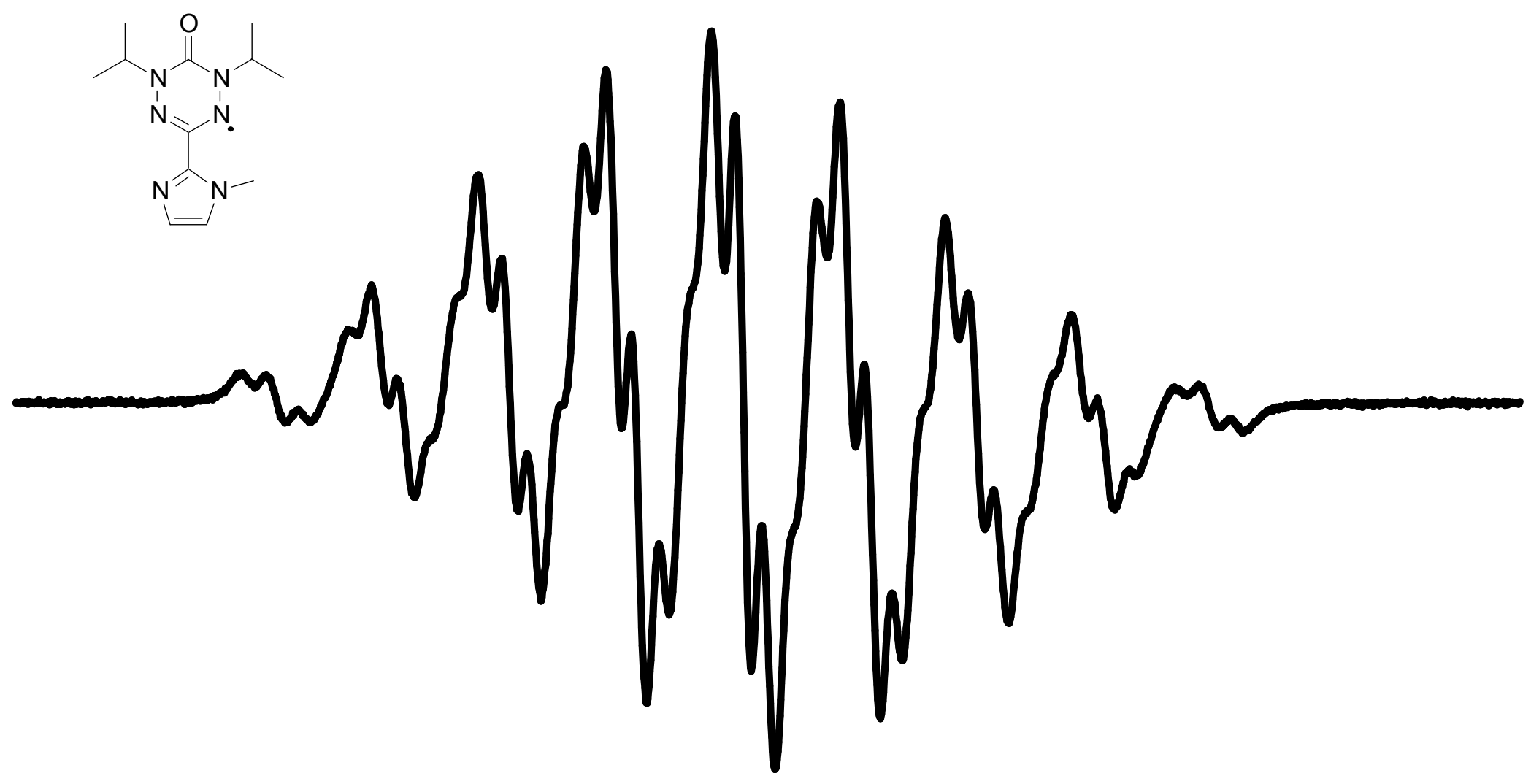

3460

3480

3500

3520

3540

Field (G)

Figure S41. EPR Spectrum of $\mathbf{2} \mathbf{f}$ in degassed $\mathrm{CH}_{2} \mathrm{Cl}_{2}(\mathrm{~g}=2.0044)$ 


\section{References:}

1. Katritzky, A. R.; S. A. Belyakov; D. Cheng; H. D. Durst, Synthesis 1995, 577.

2. Neugebauer, F. A.; H. Trischmann; G. Taigel, Monatsh. Chem. 1967, 98, 713.

3. Gilroy, J. B.; M. J. Ferguson; R. McDonald; B. O. Patrick; R. G. Hicks, Chem. Commun. 2007, 126.

4. Ibrahim, Y. A.; A. H. M. Elwahy; A. A. Abbas, Tetrahedron 1994, 50, 11489.

5. Kuhn, R.; H. Trischmann, Monatsh. Chem. 1964, 95, 457.

6. Mukai, K.; T. Yamamoto; M. Kohno; N. Azuma; K. Ishizu, Bull. Chem. Soc. Jpn. 1974, 47, 1797.

7. Neugebauer, F. A.; H. Brunner, Tetrahedron 1974, 30, 2841.

8. Mukai, K.; M. Matsubara; H. Hisatou; Y. Hosokoshi; K. Inoue; N. Azuma, J. Phys. Chem. B 2002, $106,8632$.

9. Neugebauer, F. A.; H. Fischer; R. Siegel, Angew. Chem. Int. Ed. 1980, 19, 724.

10. Gilroy, J. B.; S. D. J. McKinnon; P. Kennepohl; M. S. Zsombor; M. J. Ferguson; L. K. Thompson; R. G. Hicks, J. Org. Chem. 2007, in Press.

11. Pare, E.C; D. J. R. Brook; A. Brieger; M. Badik; M. Schinke, Org. Biomol. Chem. 2005, 3, 4258. 12. Milcent, R.; G. Barbier; S. Capelle; J. P. Catteau, J. Hetrocycl. Chem. 1994, 31, 319. 